\title{
Fractional Hermite-Jensen-Mercer Integral Inequalities with respect to Another Function and Application
}

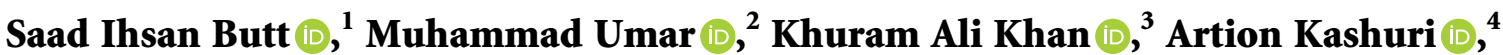 \\ and Homan Emadifar $\mathbb{D i D}^{5}$ \\ ${ }^{1}$ COMSATs University Islamabad, Lahore Campus, Lahore, Pakistan \\ ${ }^{2}$ Afro-Asian Institute, Lahore, Pakistan \\ ${ }^{3}$ Department of Mathematics, University of Sargdha, Sargodha 40100, Pakistan \\ ${ }^{4}$ Department of Mathematics, Faculty of Technical Science, University "Ismail Qemali", 9400 Vlorë, Albania \\ ${ }^{5}$ Department of Mathematics, Islamic Azad University, Hamedan Branch, Hamedan, Iran
}

Correspondence should be addressed to Homan Emadifar; homan_emadi@yahoo.com

Received 18 June 2021; Accepted 18 August 2021; Published 6 September 2021

Academic Editor: Muhammad Imran Asjad

Copyright (c) 2021 Saad Ihsan Butt et al. This is an open access article distributed under the Creative Commons Attribution License, which permits unrestricted use, distribution, and reproduction in any medium, provided the original work is properly cited.

In this paper, authors prove new variants of Hermite-Jensen-Mercer type inequalities using $\psi$-Riemann-Liouville fractional integrals with respect to another function via convexity. We establish generalized identities involving $\psi$-Riemann-Liouville fractional integral pertaining first and twice differentiable convex function $\lambda$, and these will be used to derive novel estimates for some fractional Hermite-Jensen-Mercer type inequalities. Some known results are recaptured from our results as special cases. Finally, an application from our results using the modified Bessel function of the first kind is established as well.

\section{Introduction and Preliminaries}

The theory of fractional integrals and derivatives has occurred in many fields and directions such as partial differential equations, difference equations, probability, and stochastic processes (see [1-6]). Behind it, the theory of convex functions with integral inequalities is also useful.

Definition 1. A function $\lambda: J \subset \Re \longrightarrow \Re$ is said to be convex on $J$ if

$$
\lambda\left((1-\zeta) y_{1}+\zeta y_{2}\right) \leq(1-\zeta) \lambda\left(y_{1}\right)+\zeta \lambda\left(y_{2}\right),
$$

holds for every $y_{1}, y_{2} \in J$ and $\zeta \in[0,1]$.

One of the best-known inequalities for convex functions is the following Hermite-Hadamard's inequality: if $\lambda: J \subseteq \Re \longrightarrow \Re$ is a convex function in $J$, where $y_{1}, y_{2} \in J$ and $y_{1}<y_{2}$, then

$$
\lambda\left(\frac{y_{1}+y_{2}}{2}\right) \leq \frac{1}{y_{2}-y_{1}} \int_{y_{1}}^{y_{2}} \lambda(\zeta) \mathrm{d} \zeta \leq \frac{\lambda\left(y_{1}\right)+\lambda\left(y_{2}\right)}{2}(\mathrm{H}-\mathrm{H})
$$

It is also known as classical $(\mathrm{H}-\mathrm{H})$ inequality. A number of mathematicians in the field of applied and pure mathematics have dedicated their efforts to extend, generalize, counterpart, and refine Hermite-Hadamard's inequality $(\mathrm{H}-\mathrm{H})$ for different classes of convex functions. For more recent results obtained on inequality $(\mathrm{H}-\mathrm{H})$, we refer the reader to references [7-10].

Let $\sigma_{i} \in[0,1]$ be nonnegative weights such that $\sum_{i=1}^{n} \sigma_{i}=1$. The Jensen inequality states that, if $\lambda$ is convex function on $\left[y_{1}, y_{2}\right]$, then

$$
\lambda\left(\sum_{i=1}^{n} \sigma_{i} \ell_{i}\right) \leq \sum_{i=1}^{n} \sigma_{i} \lambda\left(\ell_{i}\right),
$$

holds for all $\ell_{i} \in\left[y_{1}, y_{2}\right]$ and all $i=1,2, \ldots, n$, see [11]. 
In the literature, Jensen's inequality and Hermite-Hadamard's inequality are highly familiar results pertaining convex functions. One of the well-known and most significant inequalities in mathematical analysis is Jensen's and related inequalities. Jensen's inequality for differentiable convex functions plays a significant role in the field of inequalities as several other inequalities can be seen as special cases of it. It is used in order to make claims regarding the function while just a little is known or is needed to be known about the distribution. Furthermore, this inequality has been used in various areas of sciences and technology to solve several problems, such as engineering, mathematical statistics, financial economics, and computer science. Some recent results can be seen in [12-14].

Jensen's inequality has a following variant gave by Mercer (see [15]).

Theorem 1. Let $\lambda$ be a convex function on $\left[y_{1}, y_{2}\right]$, then

$$
\lambda\left(y_{1}+y_{2}-\sum_{i=1}^{n} \sigma_{i} \ell_{i}\right) \leq \lambda\left(y_{1}\right)+\lambda\left(y_{2}\right)-\sum_{i=1}^{n} \sigma_{i} \lambda\left(\ell_{i}\right)
$$

holds for all $\ell_{i} \in\left[y_{1}, y_{2}\right]$ and all $\sigma_{i} \in[0,1],(i=1,2, \ldots, n)$.

Jensen-Mercer's type inequality is a topic of supreme interest as it gives more information with explicit boundary conditions. It is quite effective for applications in operator analysis in higher dimensions [16-18]. Moradi et al. established some new improvements and generalization of Jensen-Mercer's type inequalities [19]. Recently, in [20], Adil et al. gave applications of Jensen-Mercer's inequality in information theory. They computed new estimates for Csiszár and related divergences. Taking into consideration the wonderful packages of Jensen's and associated inequalities in diverse fields of mathematics and engineering sciences, their generalizations and upgrades were a subject of an excellent hobby for the researchers in the last few years as obvious from a massive variety of investigation on it (see [21-24]).

In [25], Vanterler da Costa Sousa and Capelas de Oliveira introduced $\psi$-fractional integrals and $\psi$-Hilfer fractional derivative with respect to another function. They also studied Gronwall inequalities using $\psi$-Hilfer operator (see [26]).

Definition 2 (see [23]). Suppose that $\left(y_{1}, y_{2}\right)\left(-\infty \leq y_{1}<\right.$ $\left.y_{2} \leq \infty\right)$ and $\alpha>0$. Also let $\psi$ be an increasing and positive monotone function on $\left(y_{1}, y_{2}\right]$, having a continuous derivative $\psi^{\prime}$ on $\left(y_{1}, y_{2}\right)$. Then, the left-sided and right-sided $\psi$-Riemann-Liouville fractional integrals of a function $\lambda$ with respect to another function $\psi$ on $\left[y_{1}, y_{2}\right]$ are defined as follows:

$$
\begin{array}{ll}
\left(I_{y_{1}+}^{\alpha: \psi}\right) \lambda(\ell)=\frac{1}{\Gamma(\alpha)} \int_{y_{1}}^{\ell} \psi^{\prime}(\zeta)(\psi(\ell)-\psi(\zeta))^{\alpha-1} \lambda(\zeta) \mathrm{d} \zeta, & y_{1}<\ell, \\
\left(I_{y_{2}-}^{\alpha: \psi}\right) \lambda(\ell)=\frac{1}{\Gamma(\alpha)} \int_{\ell}^{y_{2}} \psi^{\prime}(\zeta)(\psi(\zeta)-\psi(\ell))^{\alpha-1} \lambda(\zeta) \mathrm{d} \zeta, & \ell<y_{2},
\end{array}
$$

respectively.

If we choose $\psi(\zeta)=\zeta$ and $\psi(\zeta)=\ln \zeta$, then we get, respectively, Riemann-Liouville and Hadamard fractional integrals.

Motivated by previous results, we will establish several new Hermite-Hadamard-Mercer type inequalities involving $\psi$-Riemann-Liouville fractional integrals (i.e., Riemann-Liouville fractional integral of any function with respect to another function). Moreover, our results recover several known results. Finally, an application using the modified Bessel function of the first kind will be established as well.

\section{Hermite-Jensen-Mercer Type Inequalities}

Throughout the paper, the following assumption will be used in the sequel.

$\left(A_{1}\right)$ : Let $0 \leq y_{1}<y_{2}, \lambda:\left[y_{1}, y_{2}\right] \longrightarrow \Re$ be a positive function and $\lambda \in L_{1}\left[y_{1}, y_{2}\right]$. Also suppose, $\psi(\cdot)$ is an increasing and positive monotone function on $\left(y_{1}, y_{2}\right]$, having a continuous derivative $\psi^{\prime}$ on $\left(y_{1}, y_{2}\right)$ and $\alpha>0$.

Theorem 2. If $\left(A_{1}\right)$ is satisfied and $\lambda$ is a convex function on $\left[y_{1}, y_{2}\right]$, then

$$
\begin{aligned}
\lambda\left(y_{1}+y_{2}-\frac{\ell_{1}}{+} \ell_{2} 2\right) \leq & {\left[\lambda\left(y_{1}\right)+\lambda\left(y_{2}\right)\right]-\frac{\Gamma(\alpha+1)}{2\left(\ell_{2}-\ell_{1}\right)^{\alpha}} } \\
& \times\left\{\left(I_{\psi^{-1}\left(\ell_{1}\right)^{+}}^{\alpha: \psi}\right)(\lambda \circ \psi)\left(\psi^{-1}\left(\ell_{2}\right)\right)+\left(I_{\psi^{-1}\left(\ell_{2}\right)^{-}}^{\alpha: \psi}(\lambda \circ \psi)\left(\psi^{-1}\left(\ell_{1}\right)\right)\right\}\right. \\
\leq & {\left[\lambda\left(y_{1}\right)+\lambda\left(y_{2}\right)\right]-\lambda\left(\frac{\ell_{1}+\ell_{2}}{2}\right), }
\end{aligned}
$$




$$
\begin{aligned}
\lambda\left(y_{1}+y_{2}-\frac{\ell_{1}+\ell_{2}}{2}\right) \leq & \frac{2^{\alpha-1} \Gamma(\alpha+1)}{\left(\ell_{2}-\ell_{1}\right)^{\alpha}} \\
& \times\left\{\left(I_{\psi^{-1}\left(y_{1}+y_{2}-\ell_{2}\right)^{+}}^{\alpha: \psi}(\lambda \circ \psi)\left(\psi^{-1}\left(y_{1}+y_{2}-\ell_{1}\right)\right)\right.\right. \\
& +\left(I_{\psi^{-1}\left(y_{1}+y_{2}-\ell_{1}\right)^{-}}^{\alpha: \psi}(\lambda \circ \psi)\left(\psi^{-1}\left(y_{1}+y_{2}-\ell_{2}\right)\right)\right\} \\
\leq & \lambda\left(y_{1}\right)+\lambda\left(y_{2}\right)-\left(\frac{\lambda\left(\ell_{1}\right)+\lambda\left(\ell_{2}\right)}{2}\right),
\end{aligned}
$$

for all $\ell_{1}, \ell_{2} \in\left[y_{1}, y_{2}\right]$, where $\Gamma(\cdot)$ is the gamma function.

Proof. Using Jensen-Mercer's inequality, we have

$$
\lambda\left(y_{1}+y_{2}-\frac{x+z}{2}\right) \leq \lambda\left(y_{1}\right)+\lambda\left(y_{2}\right)-\frac{\lambda(x)+\lambda(z)}{2},
$$

for all $x, z \in\left[y_{1}, y_{2}\right]$.

Now, by change of variables $x=\zeta \ell_{1}+(1-\zeta) \ell_{2}$ and $z=(1-\zeta) \ell_{1}+\zeta \ell_{2}$, for all $\ell_{1}, \ell_{2} \in\left[y_{1}, y_{2}\right]$ and $\zeta \in[0,1]$ in (8), we get

$$
\lambda\left(y_{1}+y_{2}-\frac{\ell_{1}+\ell_{2}}{2}\right) \leq \lambda\left(y_{1}\right)+\lambda\left(y_{2}\right)-\frac{\lambda\left(\zeta \ell_{1}+(1-\zeta) \ell_{2}\right)+\lambda\left((1-\zeta) \ell_{1}+\zeta \ell_{2}\right)}{2}
$$

Multiplying the above inequality by $\zeta^{\alpha-1}$ on both sides and integrating with respect to $\zeta$ on $[0,1]$, we obtain

$$
\begin{aligned}
\frac{1}{\alpha} \lambda\left(y_{1}+y_{2}-\frac{\ell_{1}+\ell_{2}}{2}\right) \leq & \frac{1}{\alpha}\left\{\lambda\left(y_{1}\right)+\lambda\left(y_{2}\right)\right\} \\
& -\frac{1}{2}\left\{\int_{0}^{1} \zeta^{\alpha-1}\left(\lambda\left(\zeta \ell_{1}+(1-\zeta) \ell_{2}\right)+\lambda\left((1-\zeta) \ell_{1}+\zeta \ell_{2}\right)\right) \mathrm{d} \zeta\right\}
\end{aligned}
$$

where

$$
\begin{aligned}
& \frac{\alpha}{2}\left\{\int_{0}^{1} \zeta^{\alpha-1}\left(\lambda\left(\zeta \ell_{1}+(1-\zeta) y_{2}\right)+\lambda\left((1-\zeta) y_{1}+\zeta y_{2}\right)\right) \mathrm{d} \zeta\right\} \\
& =\frac{\alpha}{2} \int_{0}^{1} \zeta^{\alpha-1} \lambda\left(\zeta \ell_{1}+(1-\zeta) \ell_{2}\right) \mathrm{d} \zeta+\frac{\alpha}{2} \int_{0}^{1} \zeta^{\alpha-1} \lambda\left((1-\zeta) \ell_{1}+\zeta \ell_{2}\right) \mathrm{d} \zeta .
\end{aligned}
$$

Now, let $\zeta=\left(\psi(\gamma)-\ell_{1}\right) /\left(\ell_{2}-\ell_{1}\right)$, then $\mathrm{d} \zeta=\left(\psi^{\prime}(\gamma) \mathrm{d} \gamma\right)$ $/\left(\ell_{2}-\ell_{1}\right)$. Using the above equality, we obtain 


$$
\begin{aligned}
= & \frac{\alpha}{2} \int_{\psi^{-1}\left(\ell_{1}\right)}^{\psi^{-1}\left(\ell_{2}\right)}\left(\frac{\ell_{2}-\psi(\gamma)}{\ell_{2}-\ell_{1}}\right)^{\alpha-1} \lambda(\psi(\gamma)) \frac{\psi^{\prime}(\gamma)}{\ell_{2}-\ell_{1}} \mathrm{~d} \gamma \\
& +\frac{\alpha}{2} \int_{\psi^{-1}\left(\ell_{1}\right)}^{\psi^{-1}\left(\ell_{2}\right)}\left(\frac{\psi(\gamma)-\ell_{1}}{\ell_{2}-\ell_{1}}\right)^{\alpha-1} \lambda(\psi(\gamma)) \frac{\psi^{\prime}(\gamma)}{\ell_{2}-\ell_{1}} \mathrm{~d} \gamma \\
= & \frac{\Gamma(\alpha+1)}{2\left(\ell_{2}-\ell_{1}\right)^{\alpha}} \frac{1}{\Gamma(\alpha)}\left\{\int_{\psi^{-1}\left(\ell_{1}\right)}^{\psi^{-1}\left(\ell_{2}\right)} \psi^{\prime}(\gamma)\left(\ell_{2}-\psi(\gamma)\right)^{\alpha-1}(\lambda \circ \psi)(\gamma) \mathrm{d} \gamma\right\} \\
& +\frac{\Gamma(\alpha+1)}{2\left(\ell_{2}-\ell_{1}\right)^{\alpha}} \frac{1}{\Gamma(\alpha)}\left\{\int_{\psi^{-1}\left(\ell_{1}\right)}^{\psi^{-1}\left(\ell_{2}\right)} \psi^{\prime}(\gamma)\left(\psi(\gamma)-\ell_{1}\right)^{\alpha-1}(\lambda \circ \psi)(\gamma) \mathrm{d} \gamma\right\} .
\end{aligned}
$$

So, the final form will be of this type as follows:

$$
\begin{aligned}
= & \frac{\Gamma(\alpha+1)}{2\left(\ell_{2}-\ell_{1}\right)^{\alpha}} \\
& \times\left\{\left(I_{\psi^{-1}\left(\ell_{1}\right)^{+}}^{\alpha: \psi}\right)(\lambda \circ \psi)\left(\psi^{-1}\left(\ell_{2}\right)\right)+\left(I_{\psi^{-1}\left(\ell_{2}\right)^{-}}^{\alpha: \psi}\right)(\lambda \circ \psi)\left(\psi^{-1}\left(\ell_{1}\right)\right)\right\},
\end{aligned}
$$

and so the first inequality of (6) is proved.

Regarding the second inequality of (6), since $\lambda$ is convex function, then for $\zeta \in[0,1]$, we have

$$
\begin{aligned}
\lambda\left(\frac{\ell_{1}+\ell_{2}}{2}\right) & =\lambda\left(\frac{\zeta \ell_{1}+(1-\zeta) \ell_{2}+(1-\zeta) \ell_{1}+\zeta \ell_{2}}{2}\right) \\
& \leq \frac{\lambda\left(\zeta \ell_{1}+(1-\zeta) \ell_{2}\right)+\lambda\left((1-\zeta) \ell_{1}+\zeta \ell_{2}\right)}{2} .
\end{aligned}
$$

Multiplying the above inequality by $\zeta^{\alpha-1}$ on both sides and integrating with respect to $\zeta$ on $[0,1]$, we get

$$
\frac{1}{\alpha} \lambda\left(\frac{\ell_{1}+\ell_{2}}{2}\right) \leq \frac{1}{2}\left\{\int_{0}^{1} \zeta^{\alpha-1}\left(\lambda\left(\zeta \ell_{1}+(1-\zeta) \ell_{2}\right)+\lambda\left((1-\zeta) \ell_{1}+\zeta \ell_{2}\right)\right)\right\}
$$

Let $\psi(\gamma)=\zeta \ell_{1}+(1-\zeta) \ell_{2}$ and $\psi(\beta)=(1-\zeta) \ell_{1}+\zeta \ell_{2}$.

Then, we have

$$
\lambda\left(\frac{\ell_{1}+\ell_{2}}{2}\right) \leq \frac{\Gamma(\alpha+1)}{2\left(\ell_{2}-\ell_{1}\right)^{\alpha}}\left\{\left(I_{\psi^{-1}\left(\ell_{1}\right)^{+}}^{\alpha: \psi}\right)(\lambda \circ \psi)\left(\psi^{-1}\left(\ell_{2}\right)\right)+\left(I_{\psi^{-1}\left(\ell_{2}\right)^{-}}^{\alpha: \psi}\right)(\lambda \circ \psi)\left(\psi^{-1}\left(\ell_{1}\right)\right)\right\} .
$$

Multiplying by $(-1)$, we will get

$$
-\frac{\Gamma(\alpha+1)}{2\left(\ell_{2}-\ell_{1}\right)^{\alpha}}\left\{\left(I_{\psi^{-1}\left(\ell_{1}\right)^{+}}^{\alpha: \psi}\right)(\lambda \circ \psi)\left(\psi^{-1}\left(\ell_{2}\right)\right)+\left(I_{\psi^{-1}\left(\ell_{2}\right)^{-}}^{\alpha: \psi}\right)(\lambda \circ \psi)\left(\psi^{-1}\left(\ell_{1}\right)\right)\right\} \leq-\lambda\left(\frac{\ell_{1}+\ell_{2}}{2}\right) .
$$

Adding $\lambda\left(y_{1}\right)+\lambda\left(y_{2}\right)$ both sides in (17), we obtain our second inequality of (6).
To prove the first inequality of (7) by using the convexity of $\lambda$, we have 


$$
2 \lambda\left(y_{1}+y_{2}-\frac{x+z}{2}\right) \leq \lambda\left(y_{1}+y_{2}-x\right)+\lambda\left(y_{1}+y_{2}-z\right),
$$

Multiplying the above inequality by $\zeta^{\alpha-1}$ on both sides and integrating with respect to $\zeta$ over $[0,1]$, we get

for all $x, z \in\left[y_{1}, y_{2}\right]$. By change of variables $x=\zeta \ell_{1}+(1-$ $\zeta) \ell_{2}$ and $z=(1-\zeta) \ell_{1}+\zeta \ell_{2}, \zeta \in[0,1]$, we get

$$
\begin{aligned}
2 \lambda\left(y_{1}+y_{2}-\frac{\ell_{1}+\ell_{2}}{2}\right) \leq & \lambda\left(y_{1}+y_{2}-\left(\zeta \ell_{1}+(1-\zeta) \ell_{2}\right)\right) \\
& +\lambda\left(y_{1}+y_{2}-\left((1-\zeta) \ell_{1}+\zeta \ell_{2}\right)\right) .
\end{aligned}
$$

$$
\begin{aligned}
& \frac{2}{\alpha} \lambda\left(y_{1}+y_{2}-\frac{\ell_{1}+\ell_{2}}{2}\right) \\
& \leq \int_{0}^{1} \zeta^{\alpha-1}\left(\lambda\left(y_{1}+y_{2}-\left(\zeta \ell_{1}+(1-\zeta) \ell_{2}\right)\right)+\lambda\left(y_{1}+y_{2}-\left((1-\zeta) \ell_{1}+\zeta \ell_{2}\right)\right)\right) \mathrm{d} \zeta
\end{aligned}
$$

Hence, by change of variables, we obtain

$$
\begin{aligned}
& \lambda\left(y_{1}+y_{2}-\frac{\ell_{1}+\ell_{2}}{2}\right) \leq \frac{2^{\alpha-1} \Gamma(\alpha+1)}{\left(\ell_{2}-\ell_{1}\right)^{\alpha}} \\
& \times\left\{\left(I_{\psi^{-1}\left(y_{1}+y_{2}-\ell_{2}\right)^{+}}^{\alpha:}\right)(\lambda \circ \Psi)\left(\psi^{-1}\left(y_{1}+y_{2}-\ell_{1}\right)\right)+\left(I_{\psi^{-1}}^{\alpha: \psi}\left(y_{1}+y_{2}-\ell_{1}\right)^{-}\right)(\lambda \circ \psi)\left(\psi^{-1}\left(y_{1}+y_{2}-\ell_{2}\right)\right)\right\},
\end{aligned}
$$

and so the first inequality of (7) is proved.

About the second inequality of (7), since $\lambda$ is convex function, then for $\zeta \in[0,1]$, we obtain

$$
\begin{aligned}
& \lambda\left(y_{1}+y_{2}-\left(\zeta \ell_{1}+(1-\zeta) \ell_{2}\right)\right) \leq \lambda\left(y_{1}\right)+\lambda\left(y_{2}\right)-\left[\zeta \lambda\left(\ell_{1}\right)+(1-\zeta) \lambda\left(\ell_{2}\right)\right], \\
& \lambda\left(y_{1}+y_{2}-\left((1-\zeta) \ell_{1}+\zeta \ell_{2}\right)\right) \leq \lambda\left(y_{1}\right)+\lambda\left(y_{2}\right)-\left[(1-\zeta) \lambda\left(\ell_{1}\right)+\zeta \lambda\left(\ell_{2}\right)\right] .
\end{aligned}
$$

By adding inequalities (22) and (23), we have

$$
\begin{aligned}
& \lambda\left(y_{1}+y_{2}-\left(\zeta \ell_{1}+(1-\zeta) \ell_{2}\right)\right)+\lambda\left(y_{1}+y_{2}-\left((1-\zeta) \ell_{1}+\zeta \ell_{2}\right)\right) \\
& \quad \leq 2\left(\lambda\left(y_{1}\right)+\lambda\left(y_{2}\right)\right)-\left(\lambda\left(\ell_{1}\right)+\lambda\left(\ell_{2}\right)\right) .
\end{aligned}
$$

Multiplying the above inequality by $\zeta^{\alpha-1}$ on both sides and integrating with respect to $\zeta$ on $[0,1]$, we get

$$
\begin{aligned}
& \frac{2^{\alpha} \Gamma(\alpha)}{\left(\ell_{2}-\ell_{1}\right)^{\alpha}}\left\{\left(I_{\psi^{-1}\left(y_{1}+y_{2}-\ell_{2}\right)^{+}}^{\alpha: \psi}\right)(\lambda \circ \psi)\left(\psi^{-1}\left(y_{1}+y_{2}-\ell_{1}\right)\right)+\left(I_{\psi^{-1}\left(y_{1}+y_{2}-\ell_{1}\right)^{-}}^{\alpha: \psi}\right)(\lambda \circ \psi)\left(\psi^{-1}\left(y_{1}+y_{2}-\ell_{2}\right)\right)\right\} \\
& \leq\left\{2\left(\lambda\left(y_{1}\right)+\lambda\left(y_{2}\right)\right)-\left(\lambda\left(\ell_{1}\right)+\lambda\left(\ell_{2}\right)\right)\right\} \cdot \frac{1}{\alpha} .
\end{aligned}
$$


Multiplying by $\alpha / 2$, we will get

$$
\begin{aligned}
& \frac{2^{\alpha-1} \Gamma(\alpha+1)}{\left(\ell_{2}-\ell_{1}\right)^{\alpha}}\left\{\left(I_{\psi^{-1}\left(y_{1}+y_{2}-\ell_{2}\right)^{+}}^{\alpha: \psi}(\lambda \circ \psi)\left(\psi^{-1}\left(y_{1}+y_{2}-\ell_{1}\right)\right)+\left(I_{\psi^{-1}\left(y_{1}+y_{2}-\ell_{1}\right)^{-}}^{\alpha: \psi}(\lambda \circ \psi)\left(\psi^{-1}\left(y_{1}+y_{2}-\ell_{2}\right)\right)\right\}\right.\right. \\
& \leq\left(\lambda\left(y_{1}\right)+\lambda\left(y_{2}\right)\right)-\frac{\lambda\left(\ell_{1}\right)+\lambda\left(\ell_{2}\right)}{2} .
\end{aligned}
$$

From inequalities (21) and (26), we get the desired double inequality (7).

Remark 1. Taking $\psi(\gamma)=\gamma$ in Theorem 2, we will get Theorem 2 proved in [27].

Remark 2. Taking $\psi(\gamma)=\gamma$ and $\alpha=1$ in Theorem 2, we will obtain Theorem 2 proved by Kian and Moslehian in [28].

Theorem 3. If $\left(A_{1}\right)$ is satisfied and $\lambda$ is a convex function on $\left[y_{1}, y_{2}\right]$, then

$$
\begin{aligned}
& \lambda\left(y_{1}+y_{2}-\frac{\ell_{1}+\ell_{2}}{2}\right) \leq \frac{2^{\alpha-1} \Gamma(\alpha+1)}{\left(\ell_{2}-\ell_{1}\right)^{\alpha}} \\
& \quad \times\left\{\left(I_{\psi^{-1}\left(y_{1}+y_{2}-\left(\left(\ell_{1}+\ell_{2}\right) / 2\right)\right)^{+}}^{\alpha: \psi}(\lambda \circ \psi)\left(\psi^{-1}\left(y_{1}+y_{2}-\ell_{1}\right)\right)\right.\right. \\
& \quad+\left(I_{\psi^{-1}\left(y_{1}+y_{2}-\left(\left(\ell_{1}+\ell_{2}\right) / 2\right)\right)^{-}}^{\alpha: \psi}(\lambda \circ \psi)\left(\psi^{-1}\left(y_{1}+y_{2}-\ell_{2}\right)\right)\right\} \\
& \leq \lambda\left(y_{1}\right)+\lambda\left(y_{2}\right)-\left(\frac{\lambda\left(\ell_{1}\right)+\lambda\left(\ell_{2}\right)}{2}\right),
\end{aligned}
$$

for all $\ell_{1}, \ell_{2} \in\left[y_{1}, y_{2}\right]$.

Proof. About the first inequality (27) by using the convexity of $\lambda$, we have

$$
2 \lambda\left(y_{1}+y_{2}-\frac{x+z}{2}\right) \leq \lambda\left(y_{1}+y_{2}-x\right)+\lambda\left(y_{1}+y_{2}-z\right),
$$

for all $x, z \in\left[y_{1}, y_{2}\right]$. By change of variables $x=(\zeta / 2) \ell_{1}+$ $((2-\zeta) / 2) \ell_{2}$ and $z=((2-\zeta) / 2) \ell_{1}+(\zeta / 2) \ell_{2}, \zeta \in[0,1]$, we get

$$
\begin{aligned}
& 2 \lambda\left(y_{1}+y_{2}-\frac{\ell_{1}+\ell_{2}}{2}\right) \leq \lambda\left(y_{1}+y_{2}-\left(\frac{\zeta}{2} \ell_{1}+\frac{2-\zeta}{2} \ell_{2}\right)\right) \\
& \quad+\lambda\left(y_{1}+y_{2}-\left(\frac{2-\zeta}{2} \ell_{1}+\frac{\zeta}{2} \ell_{2}\right)\right) .
\end{aligned}
$$

Multiplying the above inequality by $\zeta^{\alpha-1}$ on both sides and integrating with respect to $\zeta$ over $[0,1]$, we have

$$
\begin{aligned}
& \frac{2}{\alpha} \lambda\left(y_{1}+y_{2}-\frac{\ell_{1}+\ell_{2}}{2}\right) \\
& \leq \int_{0}^{1} \zeta^{\alpha-1}\left(\lambda\left(y_{1}+y_{2}-\left(\frac{\zeta}{2} \ell_{1}+\frac{2-\zeta}{2} \ell_{2}\right)\right)+\lambda\left(y_{1}+y_{2}-\left(\frac{2-\zeta}{2} \ell_{1}+\frac{\zeta}{2} \ell_{2}\right)\right)\right) \mathrm{d} \zeta .
\end{aligned}
$$

Hence, by change of variables, we obtain

$$
\begin{aligned}
\lambda\left(y_{1}+y_{2}-\frac{\ell_{1}+\ell_{2}}{2}\right) \leq & \frac{2^{\alpha-1} \Gamma(\alpha+1)}{\left(\ell_{2}-\ell_{1}\right)^{\alpha}} \\
& \times\left\{\left(I_{\psi^{-1}\left(y_{1}+y_{2}-\left(\left(\ell_{1}+\ell_{2}\right) / 2\right)\right)^{+}}^{\psi_{*}}\right)(\lambda \circ \Psi)\left(\psi^{-1}\left(y_{1}+y_{2}-\ell_{1}\right)\right)+\left(I_{\psi^{-1}\left(y_{1}+y_{2}-\left(\left(\ell_{1}+\ell_{2}\right) / 2\right)\right)^{-}}^{\alpha: \psi}(\lambda \circ \psi)\left(\psi^{-1}\left(y_{1}+y_{2}-\ell_{2}\right)\right)\right\},\right.
\end{aligned}
$$

which proved the first inequality of (27).

Regarding the second inequality of (27), since $\lambda$ is convex function, then for $\zeta \in[0,1]$, we have 


$$
\begin{aligned}
& \lambda\left(y_{1}+y_{2}-\left(\frac{\zeta}{2} \ell_{1}+\frac{2-\zeta}{2} \ell_{2}\right)\right) \leq \lambda\left(y_{1}\right)+\lambda\left(y_{2}\right)-\left[\frac{\zeta}{2} \lambda\left(\ell_{1}\right)+\frac{2-\zeta}{2} \lambda\left(\ell_{2}\right)\right] \\
& \lambda\left(y_{1}+y_{2}-\left(\frac{2-\zeta}{2} \ell_{1}+\frac{\zeta}{2} \ell_{2}\right)\right) \leq \lambda\left(y_{1}\right)+\lambda\left(y_{2}\right)-\left[\frac{2-\zeta}{2} \lambda\left(\ell_{1}\right)+\frac{\zeta}{2} \lambda\left(\ell_{2}\right)\right] .
\end{aligned}
$$

By adding inequalities (32) and (33), we get

$$
\begin{aligned}
& \lambda\left(y_{1}+y_{2}-\left(\frac{\zeta}{2} \ell_{1}+\frac{2-\zeta}{2} \ell_{2}\right)\right)+\lambda\left(y_{1}+y_{2}-\left(\frac{2-\zeta}{2} \ell_{1}+\frac{\zeta}{2} \ell_{2}\right)\right) \\
& \leq 2\left(\lambda\left(y_{1}\right)+\lambda\left(y_{2}\right)\right)-\left(\lambda\left(\ell_{1}\right)+\lambda\left(\ell_{2}\right)\right) .
\end{aligned}
$$

Multiplying the above inequality by $\zeta^{\alpha-1}$ on both sides and integrating with respect to $\zeta$ on $[0,1]$, we obtain

$$
\begin{aligned}
& \int_{0}^{1} \zeta^{\alpha-1}\left(\lambda\left(y_{1}+y_{2}-\left(\frac{\zeta}{2} \ell_{1}+\frac{2-\zeta}{2} \ell_{2}\right)\right)+\lambda\left(y_{1}+y_{2}-\left(\frac{2-\zeta}{2} \ell_{1}+\frac{\zeta}{2} \ell_{2}\right)\right)\right) \mathrm{d} \zeta \\
& \leq\left(2\left(\lambda\left(y_{1}\right)+\lambda\left(y_{2}\right)\right)-\left(\lambda\left(\ell_{1}\right)+\lambda\left(\ell_{2}\right)\right)\right) \int_{0}^{1} \zeta^{\alpha-1} \mathrm{~d} \zeta .
\end{aligned}
$$

Then, we have the following inequality:

$$
\begin{aligned}
& \frac{2^{\alpha} \Gamma(\alpha)}{\left(\ell_{2}-\ell_{1}\right)^{\alpha}}\left\{\left(I_{\psi^{-1}\left(y_{1}+y_{2}-\left(\left(\ell_{1}+\ell_{2}\right) / 2\right)\right)^{+}}^{\alpha: \psi}\right)(\lambda \circ \psi)\left(\psi^{-1}\left(y_{1}+y_{2}-\ell_{1}\right)\right)+\left(I_{\psi^{-1}\left(y_{1}+y_{2}-\left(\left(\ell_{1}+\ell_{2}\right) / 2\right)\right)^{-}}^{\alpha: \psi}\right)(\lambda \circ \psi)\left(\psi^{-1}\left(y_{1}+y_{2}-\ell_{2}\right)\right)\right\} \\
& \leq\left(2\left(\lambda\left(y_{1}\right)+\lambda\left(y_{2}\right)\right)-\left(\lambda\left(\ell_{1}\right)+\lambda\left(\ell_{2}\right)\right)\right) \cdot \frac{1}{\alpha}
\end{aligned}
$$

Multiplying by $\alpha / 2$, we will get

$$
\begin{aligned}
& \frac{2^{\alpha-1} \Gamma(\alpha+1)}{\left(\ell_{2}-\ell_{1}\right)^{\alpha}}\left\{\left(I_{\psi^{-1}\left(y_{1}+y_{2}-\left(\left(\ell_{1}+\ell_{2}\right) / 2\right)\right)^{+}}^{\alpha: \psi}(\lambda \circ \psi)\left(\psi^{-1}\left(y_{1}+y_{2}-\ell_{1}\right)\right)+\left(I_{\psi^{-1}\left(y_{1}+y_{2}-\left(\left(\ell_{1}+\ell_{2}\right) / 2\right)\right)^{-}}^{\alpha: \psi}(\lambda \circ \psi)\left(\psi^{-1}\left(y_{1}+y_{2}-\ell_{2}\right)\right)\right\}\right.\right. \\
& \leq\left(\lambda\left(y_{1}\right)+\lambda\left(y_{2}\right)\right)-\frac{\lambda\left(\ell_{1}\right)+\lambda\left(\ell_{2}\right)}{2} .
\end{aligned}
$$

From inequalities (31) and (37), we get the desired double inequality (27).

Remark 3. Taking $\psi(\gamma)=\gamma$ in Theorem 3, we will get Theorem 3 proved in [27].
Remark 4. Taking $\psi(\gamma)=\gamma$ and $\alpha=1$ in Theorem 3, we will obtain Theorem 2.1 proved by Kian and Moslehian in [28].

Theorem 4. If $\left(A_{1}\right)$ is satisfied and $\lambda$ is a convex function on $\left[y_{1}, y_{2}\right]$, then 


$$
\begin{aligned}
& \lambda\left(y_{1}+y_{2}-\frac{\ell_{1}+\ell_{2}}{2}\right) \leq \frac{2^{\alpha-1} \Gamma(\alpha+1)}{\left(\ell_{2}-\ell_{1}\right)^{\alpha}} \\
& \quad \times\left\{\left(I_{\psi^{-1}\left(y_{1}+y_{2}-\ell_{2}\right)^{+}}^{\alpha: \psi}(\lambda \circ \psi)\left(\psi^{-1}\left(y_{1}+y_{2}-\frac{\ell_{1}+\ell_{2}}{2}\right)\right)+\left(I_{\psi^{-1}\left(y_{1}+y_{2}-\ell_{1}\right)^{-}}^{\alpha: \psi}(\lambda \circ \psi)\left(\psi^{-1}\left(y_{1}+y_{2}-\frac{\ell_{1}+\ell_{2}}{2}\right)\right)\right\}\right.\right. \\
& \leq \lambda\left(y_{1}\right)+\lambda\left(y_{2}\right)-\left(\frac{\lambda\left(\ell_{1}\right)+\lambda\left(\ell_{2}\right)}{2}\right)
\end{aligned}
$$

for all $\ell_{1}, \ell_{2} \in\left[y_{1}, y_{2}\right]$.

$$
\begin{aligned}
2 \lambda\left(y_{1}+y_{2}-\frac{\ell_{1}+\ell_{2}}{2}\right) \leq & \lambda\left(y_{1}+y_{2}-\left(\frac{1+\zeta}{2} \ell_{1}+\frac{1-\zeta}{2} \ell_{2}\right)\right) \\
& +\lambda\left(y_{1}+y_{2}-\left(\frac{1-\zeta_{2}}{2} \ell_{1}+\frac{1+\zeta}{2} \ell_{2}\right)\right) .
\end{aligned}
$$

Proof. Regarding the first part of inequality (38) by using the convexity of $\lambda$, we have

$$
2 \lambda\left(y_{1}+y_{2}-\frac{x+z}{2}\right) \leq \lambda\left(y_{1}+y_{2}-x\right)+\lambda\left(y_{1}+y_{2}-z\right),
$$

for all $x, z \in\left[y_{1}, y_{2}\right]$. By change of variables $x=((1+\zeta) /$ $2) \ell_{1}+((1-\zeta) / 2) \ell_{2}$ and $z=((1-\zeta) / 2) \ell_{1}+((1+\zeta) /$ 2) $\ell_{2}, \zeta \in[0,1]$, we get

$$
\begin{aligned}
& \frac{2}{\alpha} \lambda\left(y_{1}+y_{2}-\frac{\ell_{1}+\ell_{2}}{2}\right) \\
& \leq \int_{0}^{1} \zeta^{\alpha-1}\left(\lambda\left(y_{1}+y_{2}-\left(\frac{1+\zeta}{2} \ell_{1}+\frac{1-\zeta}{2} \ell_{2}\right)\right)+\lambda\left(y_{1}+y_{2}-\left(\frac{1-\zeta}{2} \ell_{1}+\frac{1+\zeta}{2} \ell_{2}\right)\right)\right) \mathrm{d} \zeta .
\end{aligned}
$$

Hence, by change of variables, we have

$$
\begin{aligned}
\lambda\left(y_{1}+y_{2}-\frac{\ell_{1}+\ell_{2}}{2}\right) \leq & \frac{2^{\alpha-1} \Gamma(\alpha+1)}{\left(\ell_{2}-\ell_{1}\right)^{\alpha}} \times\left\{\left(I_{\psi^{-1}\left(y_{1}+y_{2}-\ell_{2}\right)^{+}}^{\alpha: \psi}(\lambda \circ \psi)\left(\psi^{-1}\left(y_{1}+y_{2}-\frac{\ell_{1}+\ell_{2}}{2}\right)\right)\right.\right. \\
& +\left(I_{\psi^{-1}\left(y_{1}+y_{2}-\ell_{1}\right)^{-}}^{\alpha: \psi}(\lambda \circ \psi)\left(\psi^{-1}\left(y_{1}+y_{2}-\frac{\ell_{1}+\ell_{2}}{2}\right)\right)\right\},
\end{aligned}
$$

Multiplying the above inequality by $\zeta^{\alpha-1}$ on both sides and integrating with respect to $\zeta \in[0,1]$, we obtain

which concludes the first inequality of (38).

About the second inequality of (38), since $\lambda$ is convex function, then for $\zeta \in[0,1]$, we get

$$
\begin{aligned}
& \lambda\left(y_{1}+y_{2}-\left(\frac{1+\zeta}{2} \ell_{1}+\frac{1-\zeta}{2} \ell_{2}\right)\right) \leq \lambda\left(y_{1}\right)+\lambda\left(y_{2}\right)-\left[\frac{1+\zeta}{2} \lambda\left(\ell_{1}\right)+\frac{1-\zeta}{2} \lambda\left(\ell_{2}\right)\right] \\
& \lambda\left(y_{1}+y_{2}-\left(\frac{1-\zeta}{2} \ell_{1}+\frac{1+\zeta}{2} \ell_{2}\right)\right) \leq \lambda\left(y_{1}\right)+\lambda\left(y_{2}\right)-\left[\frac{1-\zeta}{2} \lambda\left(\ell_{1}\right)+\frac{1+\zeta}{2} \lambda\left(\ell_{2}\right)\right] .
\end{aligned}
$$

By adding inequalities (43) and (44), we have 


$$
\begin{aligned}
& \lambda\left(y_{1}+y_{2}-\left(\frac{1+\zeta}{2} \ell_{1}+\frac{1-\zeta}{2} \ell_{2}\right)\right)+\lambda\left(y_{1}+y_{2}-\left(\frac{1-\zeta}{2} \ell_{1}+\frac{1+\zeta}{2} \ell_{2}\right)\right) \\
& \leq 2\left(\lambda\left(y_{1}\right)+\lambda\left(y_{2}\right)\right)-\left(\lambda\left(\ell_{1}\right)+\lambda\left(\ell_{2}\right)\right) .
\end{aligned}
$$

Multiplying the above inequality by $\zeta^{\alpha-1}$ on both sides and integrating with respect to $\zeta$ over $[0,1]$, we obtain

$$
\begin{aligned}
& \int_{0}^{1} \zeta^{\alpha-1}\left(\lambda\left(y_{1}+y_{2}-\left(\frac{1+\zeta}{2} \ell_{1}+\frac{1-\zeta}{2} \ell_{2}\right)\right)+\lambda\left(y_{1}+y_{2}-\left(\frac{1-\zeta}{2} \ell_{1}+\frac{1+\zeta}{2} \ell_{2}\right)\right)\right) \mathrm{d} \zeta \\
& \leq\left(2\left(\lambda\left(y_{1}\right)+\lambda\left(y_{2}\right)\right)-\left(\lambda\left(\ell_{1}\right)+\lambda\left(\ell_{2}\right)\right)\right) \int_{0}^{1} \zeta^{\alpha-1} \mathrm{~d} \zeta .
\end{aligned}
$$

Then, we have the following inequality:

$$
\begin{aligned}
& \frac{2^{\alpha} \Gamma(\alpha)}{\left(\ell_{2}-\ell_{1}\right)^{\alpha}} \\
& \quad \times\left\{\left(I_{\psi^{-1}}^{\alpha: \psi}\left(y_{1}+y_{2}-\ell_{2}\right)^{+}\right)(\lambda \circ \psi)\left(\psi^{-1}\left(y_{1}+y_{2}-\frac{\ell_{1}+\ell_{2}}{2}\right)\right)+\left(I_{\psi^{-1}\left(y_{1}+y_{2}-\ell_{1}\right)^{-}}^{\alpha: \psi}\right)(\lambda \circ \psi)\left(\psi^{-1}\left(y_{1}+y_{2}-\frac{\ell_{1}+\ell_{2}}{2}\right)\right)\right\} \\
& \leq\left(2\left(\lambda\left(y_{1}\right)+\lambda\left(y_{2}\right)\right)-\left(\lambda\left(\ell_{1}\right)+\lambda\left(\ell_{2}\right)\right)\right) \cdot \frac{1}{\alpha} .
\end{aligned}
$$

Multiplying by $\alpha / 2$, we will get

$$
\begin{aligned}
& \frac{2^{\alpha-1} \Gamma(\alpha+1)}{\left(\ell_{2}-\ell_{1}\right)^{\alpha}} \times\left\{\left(I_{\psi^{-1}\left(y_{1}+y_{2}-\ell_{2}\right)^{+}}^{\alpha:}\right)(\lambda \circ \psi)\left(\psi^{-1}\left(y_{1}+y_{2}-\frac{\ell_{1}+\ell_{2}}{2}\right)\right)+\left(I_{\psi^{-1}\left(y_{1}+y_{2}-\ell_{1}\right)^{-}}^{\alpha: \psi}\right)(\lambda \circ \psi)\left(\psi^{-1}\left(y_{1}+y_{2}-\frac{\ell_{1}+\ell_{2}}{2}\right)\right)\right\} \\
& \leq\left(\lambda\left(y_{1}\right)+\lambda\left(y_{2}\right)\right)-\frac{\lambda\left(\ell_{1}\right)+\lambda\left(\ell_{2}\right)}{2} .
\end{aligned}
$$

So, the second inequality of (38) holds.

Remark 5. Taking $\psi(\gamma)=\gamma$ in Theorem 4, we will get Theorem 2 proved in [29].

Remark 6. Taking $\psi(\gamma)=\gamma$ and $\alpha=1$ in Theorem 4, we will obtain Theorem 2.1 proved by Kian and Moslehian in [28].

\section{New Generalized Identities and Their Integral Inequalities}

In this section, the following lemmas will play a basic role in our next results.

Lemma 1. If $\left(A_{1}\right)$ is satisfied and $\lambda:\left[y_{1}, y_{2}\right] \longrightarrow \mathfrak{R}$ is a differentiable function on $L_{1}\left[y_{1}, y_{2}\right]$, then 
10

Complexity

$$
\begin{aligned}
& \frac{\lambda\left(y_{1}+y_{2}-\ell_{1}\right)+\lambda\left(y_{1}+y_{2}-\ell_{2}\right)}{2}-\frac{\Gamma(\alpha+1)}{2\left(\ell_{2}-\ell_{1}\right)^{\alpha}} \\
& \quad \times\left\{\left(I_{\psi^{-1}\left(y_{1}+y_{2}-\ell_{2}\right)^{+}}^{\alpha: \psi}\right)(\lambda \circ \psi)\left(\psi^{-1}\left(y_{1}+y_{2}-\ell_{1}\right)\right)+\left(I_{\psi^{-1}\left(y_{1}+y_{2}-\ell_{1}\right.}^{\alpha: \psi}\right)(\lambda \circ \psi)\left(\psi^{-1}\left(y_{1}+y_{2}-\ell_{2}\right)\right)\right\} \\
& =\frac{1}{2\left(\ell_{2}-\ell_{1}\right)^{\alpha}} \\
& \quad \times \int_{\psi^{-1}\left(y_{1}+y_{2}-\ell_{2}\right)}^{\psi^{-1}\left(y_{1}+y_{2}-\ell_{1}\right)}\left(\left(\psi(\gamma)-\left(y_{1}+y_{2}-\ell_{2}\right)\right)^{\alpha}-\left(\left(y_{1}+y_{2}-\ell_{1}\right)-\psi(\gamma)\right)^{\alpha}\right) \times\left(\lambda^{\prime} \circ \psi\right)(\gamma) \psi^{\prime}(\gamma) \mathrm{d} \gamma,
\end{aligned}
$$

for all $\ell_{1}, \ell_{2} \in\left[y_{1}, y_{2}\right]$.

$$
I=\frac{\lambda\left(y_{1}+y_{2}-\ell_{1}\right)-\lambda\left(y_{1}+y_{2}-\ell_{2}\right)}{2}-\left\{I_{1}+I_{2}\right\},
$$

Proof. It suffices to note that

where

$$
\begin{aligned}
& I_{1}=\frac{\Gamma(\alpha+1)}{2\left(\ell_{2}-\ell_{1}\right)^{\alpha}}\left[I_{\Psi^{-1}\left(y_{1}+y_{2}-\ell_{2}\right)^{+}}^{\alpha: \psi}(\lambda \circ \psi)\left(\psi^{-1}\left(y_{1}+y_{2}-\ell_{1}\right)\right)\right] \\
& =\frac{\alpha}{2\left(\ell_{2}-\ell_{1}\right)^{\alpha}} \int_{\psi^{-1}\left(y_{1}+y_{2}-\ell_{2}\right)}^{\psi^{-1}\left(y_{1}+y_{2}-\ell_{1}\right)} \psi^{\prime}(\gamma)\left(\left(y_{1}+y_{2}-\ell_{1}\right)-\psi(\gamma)\right)^{\alpha-1}(\lambda \circ \psi)(\gamma) \mathrm{d} \gamma \\
& =\frac{-1}{2\left(\ell_{2}-\ell_{1}\right)^{\alpha}} \int_{\psi^{-1}\left(y_{1}+y_{2}-\ell_{1}\right)}^{\psi^{-1}\left(y_{1}+y_{2}-\ell_{2}\right)} \mathrm{d}\left(\left(y_{1}+y_{2}-\ell_{1}\right)-\psi(\gamma)\right)^{\alpha}(\lambda \circ \psi)(\gamma) \\
& =\frac{1}{2\left(\ell_{2}-\ell_{1}\right)^{\alpha}}\left[\lambda\left(y_{1}+y_{2}-\ell_{2}\right)\left(\ell_{2}-\ell_{1}\right)^{\alpha}\right] \\
& +\int_{\psi^{-1}\left(y_{1}+y_{2}-\ell_{2}\right)}^{\psi^{-1}\left(y_{1}+y_{2}-\ell_{1}\right)} \psi^{\prime}(\gamma)\left(\left(y_{1}+y_{2}-\ell_{1}\right)-\psi(\gamma)\right)^{\alpha}\left(\lambda^{\prime} \circ \psi\right)(\gamma) \mathrm{d} \gamma, \\
& I_{2}=\frac{\Gamma(\alpha+1)}{2\left(\ell_{2}-\ell_{1}\right)^{\alpha}}\left[I_{\psi^{-1}\left(y_{1}+y_{2}-\ell_{1}\right)^{-}}^{\alpha: \psi}(\lambda \circ \psi)\left(\psi^{-1}\left(y_{1}+y_{2}-\ell_{2}\right)\right)\right] \\
& =\frac{\alpha}{2\left(\ell_{2}-\ell_{1}\right)^{\alpha}} \int_{\psi^{-1}\left(y_{1}+y_{2}-\ell_{2}\right)}^{\psi^{-1}\left(y_{1}+y_{2}-\ell_{1}\right)} \psi^{\prime}(\gamma)\left(-\left(y_{1}+y_{2}-\ell_{2}\right)+\psi(\gamma)\right)^{\alpha-1}(\lambda \circ \psi)(\gamma) \mathrm{d} \gamma \\
& =\frac{1}{2\left(\ell_{2}-\ell_{1}\right)^{\alpha}} \int_{\psi^{-1}\left(y_{1}+y_{2}-\ell_{2}\right)}^{\psi^{-1}\left(y_{1}+y_{2}-\ell_{1}\right)} \mathrm{d}\left(-\left(y_{1}+y_{2}-\ell_{2}\right)+\psi(\gamma)\right)^{\alpha}(\lambda \circ \psi)(\gamma) \\
& =\frac{1}{2\left(\ell_{2}-\ell_{1}\right)^{\alpha}}\left[\lambda\left(y_{1}+y_{2}-\ell_{1}\right)\left(\ell_{2}-\ell_{1}\right)^{\alpha}\right] \\
& -\int_{\psi^{-1}\left(y_{1}+y_{2}-\ell_{2}\right)}^{\psi^{-1}\left(y_{1}+y_{2}-\ell_{1}\right)} \psi^{\prime}(\gamma)\left(-\left(y_{1}+y_{2}-\ell_{2}\right)+\psi(\gamma)\right)^{\alpha}\left(\lambda^{\prime} \circ \psi\right)(\gamma) \mathrm{d} \gamma .
\end{aligned}
$$

Substituting (51) and (52) in (50), we get the desired Remark 7. For $\ell_{1}=y_{1}$ and $\ell_{2}=y_{2}$ in Lemma 1 , we will get equality (49). Lemma 3.1 proved in [30]. 
Theorem 5. If $\left(A_{1}\right)$ is satisfied and $\left|\lambda^{\prime}\right|$ is a convex function on $\left[y_{1}, y_{2}\right]$, then

$$
\begin{aligned}
& \mid \frac{\lambda\left(y_{1}+y_{2}-\ell_{1}\right)+\lambda\left(y_{1}+y_{2}-\ell_{2}\right)}{2}-\frac{\Gamma(\alpha+1)}{2\left(\ell_{2}-\ell_{1}\right)^{\alpha}}\left(\left(I_{\psi^{-1}\left(y_{1}+y_{2}-\ell_{2}\right)^{+}}^{\alpha: \psi}\right) \lambda\left(y_{1}+y_{2}-\ell_{1}\right)\right. \\
& \left.\quad+\left(I_{\psi^{-1}\left(y_{1}+y_{2}-\ell_{1}\right)^{-}}^{\alpha: \psi}\right) \lambda\left(y_{1}+y_{2}-\ell_{2}\right)\right) \mid \\
& \leq \frac{\left(\ell_{2}-\ell_{1}\right)}{\alpha+1}\left(1-\frac{1}{2^{\alpha}}\right)\left\{\left|\lambda^{\prime}\left(y_{1}\right)\right|+\left|\lambda^{\prime}\left(y_{2}\right)\right|-\left(\frac{\left|\lambda^{\prime}\left(\ell_{1}\right)\right|+\left|\lambda^{\prime}\left(\ell_{2}\right)\right|}{2}\right)\right\} .
\end{aligned}
$$

for all $\ell_{1}, \ell_{2} \in\left[y_{1}, y_{2}\right]$.

Proof. Here, we will use Lemma 1, properties of modulus, and Jensen-Mercer's inequality.
For every $\gamma \in\left(\psi^{-1}\left(y_{1}+y_{2}-\ell_{2}\right), \psi^{-1}\left(y_{1}+y_{2}-\ell_{1}\right)\right)$, we have $\left(y_{1}+y_{2}-\ell_{2}\right)<\psi(\gamma)<\left(y_{1}+y_{2}-\ell_{1}\right)$.

Let $\zeta=\left(\left(y_{1}+y_{2}-\ell_{1}\right)-\psi(\gamma)\right) /\left(\ell_{2}-\ell_{1}\right)$, and then $\psi(\gamma)=y_{1}+y_{2}-\left(\zeta \ell_{1}+(1-\zeta) \ell_{2}\right)$. So, we get

$$
\begin{aligned}
& \mid \frac{\lambda\left(y_{1}+y_{2}-\ell_{1}\right)+\lambda\left(y_{1}+y_{2}-\ell_{2}\right)}{2}-\frac{\Gamma(\alpha+1)}{2\left(\ell_{2}-\ell_{1}\right)^{\alpha}}\left(\left(I_{\left(y_{1}+y_{2}-\ell_{2}\right)^{+}}^{\alpha: \psi}\right) \lambda\left(y_{1}+y_{2}-\ell_{1}\right)\right. \\
& \left.+\left(I_{\left(y_{1}+y_{2}-\ell_{1}\right)^{-}}^{\alpha: \psi}\right) \lambda\left(y_{1}+y_{2}-\ell_{2}\right)\right) \mid \\
& \leq \frac{1}{2\left(\ell_{2}-\ell_{1}\right)^{\alpha}} \int_{\psi^{-1}\left(y_{1}+y_{2}-\ell_{2}\right)}^{\psi^{-1}\left(y_{1}+y_{2}-\ell_{1}\right)}\left|\left(\psi(\gamma)-\left(y_{1}+y_{2}-\ell_{2}\right)\right)^{\alpha}-\left(\left(y_{1}+y_{2}-\ell_{1}\right)-\psi(\gamma)\right)^{\alpha}\right| \times\left|\left(\lambda^{\prime} \circ \psi\right)(\gamma)\right| \psi^{\prime}(\gamma) \mathrm{d} \gamma \\
& =\frac{\left(\ell_{2}-\ell_{1}\right)}{2} \int_{0}^{1}\left|\zeta^{\alpha}-(1-\zeta)^{\alpha}\right|\left|\lambda^{\prime}\left(y_{1}+y_{2}-\left(\zeta \ell_{1}+(1-\zeta) \ell_{2}\right)\right)\right| \mathrm{d} \zeta \\
& \leq \frac{\left(\ell_{2}-\ell_{1}\right)}{2} \int_{0}^{1}\left|\zeta^{\alpha}-(1-\zeta)^{\alpha}\right|\left\{\left|\lambda^{\prime}\left(y_{1}\right)\right|+\left|\lambda^{\prime}\left(y_{2}\right)\right|-\left(\zeta\left|\lambda^{\prime}\left(\ell_{1}\right)\right|+(1-\zeta)\left|\lambda^{\prime}\left(\ell_{2}\right)\right|\right)\right\} \mathrm{d} \zeta \\
& =\frac{\left(\ell_{2}-\ell_{1}\right)}{2}\left[I_{1}+I_{2}\right] \text {, }
\end{aligned}
$$

where

$$
\begin{aligned}
I_{1}= & \int_{0}^{1 / 2}\left((1-\zeta)^{\alpha}-\zeta^{\alpha}\right)\left\{\left|\lambda^{\prime}\left(y_{1}\right)\right|+\left|\lambda^{\prime}\left(y_{2}\right)\right|-\left(\zeta\left|\lambda^{\prime}\left(\ell_{1}\right)\right|+(1-\zeta)\left|\lambda^{\prime}\left(\ell_{2}\right)\right|\right)\right\} \mathrm{d} \zeta \\
= & \left(\left|\lambda^{\prime}\left(y_{1}\right)\right|+\left|\lambda^{\prime}\left(y_{2}\right)\right|\right)\left(\frac{1}{(\alpha+1)}-\frac{2^{-\alpha}}{(\alpha+1)}\right) \\
& -\left\{\left|\lambda^{\prime}\left(\ell_{1}\right)\right|\left(\frac{1}{(\alpha+1)(\alpha+2)}-\frac{2^{-\alpha-1}}{(\alpha+1)}\right)+\left|\lambda^{\prime}\left(\ell_{2}\right)\right|\left(\frac{1}{(\alpha+2)}-\frac{2^{-\alpha-1}}{(\alpha+1)}\right)\right\}, \\
I_{2}= & \int_{1 / 2}^{1}\left(\zeta^{\alpha}-(1-\zeta)^{\alpha}\right)\left\{\left|\lambda^{\prime}\left(y_{1}\right)\right|+\left|\lambda^{\prime}\left(y_{2}\right)\right|-\left(\zeta\left|\lambda^{\prime}\left(\ell_{1}\right)\right|+(1-\zeta)\left|\lambda^{\prime}\left(\ell_{2}\right)\right|\right)\right\} \mathrm{d} \zeta \\
= & \left(\left|\lambda^{\prime}\left(y_{1}\right)\right|+\left|\lambda^{\prime}\left(y_{2}\right)\right|\right)\left(\frac{1}{(\alpha+1)}-\frac{2^{-\alpha}}{(\alpha+1)}\right) \\
& -\left\{\left|\lambda^{\prime}\left(\ell_{1}\right)\right|\left(\frac{1}{(\alpha+2)}-\frac{2^{-\alpha-1}}{(\alpha+1)}\right)+\left|\lambda^{\prime}\left(\ell_{2}\right)\right|\left(\frac{1}{(\alpha+1)(\alpha+2)}-\frac{2^{-\alpha-1}}{(\alpha+1)}\right)\right\} .
\end{aligned}
$$


Substituting (55) and (56) in (54), we get (53).

Remark 8. For $\ell_{1}=y_{1}$ and $\ell_{2}=y_{2}$ in Theorem 5, we will get Theorem 3.4 proved in [30].
Remark 9. Taking $\psi(\gamma)=\gamma$ in Theorem 5, we will get Theorem 4 proved in [27].

Lemma 2. If $\left(A_{1}\right)$ is satisfied and $\lambda:\left[y_{1}, y_{2}\right] \longrightarrow \Re$ is a differentiable function on $L_{1}\left[y_{1}, y_{2}\right]$, then

$$
\begin{aligned}
& \frac{\lambda\left(y_{1}+y_{2}-\ell_{1}\right)+\lambda\left(y_{1}+y_{2}-\ell_{2}\right)}{2}-\frac{2^{\alpha-1} \Gamma(\alpha+1)}{\left(\ell_{2}-\ell_{1}\right)^{\alpha}} \\
& \quad \times\left\{\left(I_{\psi^{-1}\left(y_{1}+y_{2}-\ell_{2}\right)^{+}}^{\alpha: \psi}\right)\left[\lambda \circ \psi\left(\psi^{-1}\left(y_{1}+y_{2}-\frac{\ell_{1}+\ell_{2}}{2}\right)\right)\right]\right. \\
& +\left(I_{\psi^{-1}\left(y_{1}+y_{2}-\ell_{1}\right)^{-}}^{\alpha: \psi}\left[\lambda \circ \psi\left(\psi^{-1}\left(y_{1}+y_{2}-\frac{\ell_{1}+\ell_{2}}{2}\right)\right)\right]\right\} \\
& =\frac{\left(\ell_{2}-\ell_{1}\right)}{4}\left[\int_{0}^{1} \zeta^{\alpha} \lambda^{\prime}\left(y_{1}+y_{2}-\left(\frac{1+\zeta}{2} \ell_{1}+\frac{1-\zeta}{2} \ell_{2}\right)\right) \mathrm{d} \zeta\right. \\
& \left.-\int_{0}^{1} \zeta^{\alpha} \lambda^{\prime}\left(y_{1}+y_{2}-\left(\frac{1-\zeta_{1}}{2} \ell_{1}+\frac{1+\zeta}{2} \ell_{2}\right)\right) \mathrm{d} \zeta\right]
\end{aligned}
$$

Proof. It suffices to note that

$$
I=\frac{\left(\ell_{2}-\ell_{1}\right)}{4}\left\{I_{1}-I_{2}\right\}
$$

$$
\begin{aligned}
& I_{1}=\int_{0}^{1} \zeta^{\alpha} \lambda^{\prime}\left(y_{1}+y_{2}-\left(\frac{1+\zeta}{2} \ell_{1}+\frac{1-\zeta}{2} \ell_{2}\right)\right) \mathrm{d} \zeta \\
& =\frac{2}{\left(\ell_{2}-\ell_{1}\right)} \lambda\left(y_{1}+y_{2}-\ell_{1}\right)-\frac{2^{\alpha}}{\ell_{2}-\ell_{1}} \int_{0}^{1} \zeta^{\alpha-1} \lambda\left(y_{1}+y_{2}-\left(\frac{1+\zeta}{2} \ell_{1}+\frac{1-\zeta}{2} \ell_{2}\right)\right) \mathrm{d} \zeta \\
& =\frac{2}{\left(\ell_{2}-\ell_{1}\right)} \lambda\left(y_{1}+y_{2}-\ell_{1}\right) \\
& -\frac{2^{\alpha+1} \Gamma(\alpha+1)}{\left(\ell_{2}-\ell_{1}\right)^{\alpha+1}}\left(I_{\psi^{-1}\left(y_{1}+y_{2}-\ell_{1}\right)^{-}}^{\alpha: \psi}\left[\lambda \circ \psi\left(\psi^{-1}\left(y_{1}+y_{2}-\frac{\ell_{1}+\ell_{2}}{2}\right)\right)\right],\right. \\
& I_{2}=\int_{0}^{1} \zeta^{\alpha} \lambda\left(y_{1}+y_{2}-\left(\frac{1-\zeta_{\ell_{1}}}{2} \frac{1+\zeta_{\ell_{2}}}{2}\right)\right) \mathrm{d} \zeta \\
& =-\frac{2}{\left(\ell_{2}-\ell_{1}\right)} \lambda\left(y_{1}+y_{2}-\ell_{2}\right)-\frac{2^{\alpha}}{\ell_{2}-\ell_{1}} \int_{0}^{1} \zeta^{\alpha-1} \lambda\left(y_{1}+y_{2}-\left(\frac{1-\zeta}{2} \ell_{1}+\frac{1+\zeta}{2} \ell_{2}\right)\right) \mathrm{d} \zeta \\
& =-\frac{2}{\left(\ell_{2}-\ell_{1}\right)} \lambda\left(y_{1}+y_{2}-\ell_{2}\right) \\
& -\frac{2^{\alpha+1} \Gamma(\alpha+1)}{\left(\ell_{2}-\ell_{1}\right)^{\alpha+1}}\left(I_{\psi^{-1}\left(y_{1}+y_{2}-\ell_{2}\right)^{+}}^{\alpha: \psi}\left[\lambda \circ \psi\left(\psi^{-1}\left(y_{1}+y_{2}-\frac{\ell_{1}+\ell_{2}}{2}\right)\right)\right]\right. \text {. }
\end{aligned}
$$

Substituting (59) and (60) in (58), we get (57).
Remark 10. Taking $\psi(\gamma)=\gamma$ in Lemma 2, we will get Lemma 1 proved in [29]. 
Theorem 6. If $\left(A_{1}\right)$ is satisfied and $\lambda^{\prime}$ is a convex function on $\left[y_{1}, y_{2}\right]$, then

$$
\begin{aligned}
& \mid \frac{\lambda\left(y_{1}+y_{2}-\ell_{1}\right)+\lambda\left(y_{1}+y_{2}-\ell_{2}\right)}{2}-\frac{2^{\alpha-1} \Gamma(\alpha+1)}{\left(\ell_{2}-\ell_{1}\right)^{\alpha}} \\
& \times\left\{\left(I_{\psi^{-1}\left(y_{1}+y_{2}-\ell_{2}\right)^{+}}^{\alpha: \psi}\left[\lambda \circ \psi\left(\psi^{-1}\left(y_{1}+y_{2}-\frac{\ell_{1}+\ell_{2}}{2}\right)\right)\right]\right.\right. \\
& +\left(I_{\psi^{-1}\left(y_{1}+y_{2}-\ell_{1}\right)^{-}}^{\alpha: \psi}\left[\lambda \circ \psi\left(\psi^{-1}\left(y_{1}+y_{2}-\frac{\ell_{1}+\ell_{2}}{2}\right)\right)\right]\right\} \mid \\
& \leq \frac{\left(\ell_{2}-\ell_{1}\right)}{4(\alpha+2)} \sup _{\xi \in\left[y_{1}, y_{2}\right]}\left|\lambda^{\prime \prime}(\xi)\right|
\end{aligned}
$$

for all $\ell_{1}, \ell_{2} \in\left[y_{1}, y_{2}\right]$.

Proof. From Lemma 2 and using mean value theorem for $\lambda^{\prime}$, we have

$$
\begin{aligned}
& \frac{\lambda\left(y_{1}+y_{2}-\ell_{1}\right)+\lambda\left(y_{1}+y_{2}-\ell_{2}\right)}{2}-\frac{2^{\alpha-1} \Gamma(\alpha+1)}{\left(\ell_{2}-\ell_{1}\right)^{\alpha}} \\
& \times\left\{\left(I_{\psi^{-1}\left(y_{1}+y_{2}-\ell_{2}\right)^{+}}^{\alpha: \psi}\right)\left[\lambda \circ \psi\left(\psi^{-1}\left(y_{1}+y_{2}-\frac{\ell_{1}+\ell_{2}}{2}\right)\right)\right]\right. \\
& \left.+\left(I_{\psi^{-1}\left(y_{1}+y_{2}-\ell_{1}\right)^{-}}^{\alpha: \psi}\right)\left[\lambda \circ \psi\left(\psi^{-1}\left(y_{1}+y_{2}-\frac{\ell_{1}+\ell_{2}}{2}\right)\right)\right]\right\} \\
& =\frac{\left(\ell_{2}-\ell_{1}\right)^{2}}{4} \int_{0}^{1} \zeta^{\alpha+1} \lambda^{\prime \prime}(\xi) \mathrm{d} \zeta,
\end{aligned}
$$

where $\xi \in\left[y_{1}, y_{2}\right]$. This leads us to

$$
\begin{aligned}
& \mid \frac{\lambda\left(y_{1}+y_{2}-\ell_{1}\right)+\lambda\left(y_{1}+y_{2}-\ell_{2}\right)}{2}-\frac{2^{\alpha-1} \Gamma(\alpha+1)}{\left(\ell_{2}-\ell_{1}\right)^{\alpha}} \\
& \quad \times\left\{\left(I_{\psi^{-1}\left(y_{1}+y_{2}-\ell_{2}\right)^{+}}^{\alpha: \psi}\right)\left[\lambda \circ \psi\left(\psi^{-1}\left(y_{1}+y_{2}-\frac{\ell_{1}+\ell_{2}}{2}\right)\right)\right]\right. \\
& \left.\quad+\left(I_{\psi^{-1}\left(y_{1}+y_{2}-\ell_{1}\right)^{-}}^{\alpha: \psi}\right)\left[\lambda \circ \psi\left(\psi^{-1}\left(y_{1}+y_{2}-\frac{\ell_{1}+\ell_{2}}{2}\right)\right)\right]\right\} \mid \\
& \leq \frac{\left(\ell_{2}-\ell_{1}\right)^{2}}{4} \int_{0}^{1}\left|\zeta^{\alpha+1}\right|\left|\lambda^{\prime \prime}(\xi)\right| \mathrm{d} \zeta \\
& \leq \frac{\left(\ell_{2}-\ell_{1}\right)^{2}}{4} \sup _{\xi \in\left[y_{1}, y_{2}\right]}\left|\lambda^{\prime \prime}(\xi)\right|\left\{\int_{0}^{1} \zeta^{\alpha+1} \mathrm{~d} \zeta\right\} \\
& =\frac{\left(\ell_{2}-\ell_{1}\right)^{2}}{4(\alpha+2)} \sup _{\xi \in\left[y_{1}, y_{2}\right]}\left|\lambda^{\prime \prime}(\xi)\right| .
\end{aligned}
$$


Remark 11. For $\psi(\gamma)=\gamma$ in Theorem 6, we will get Theorem 3 proved in [29].
Theorem 7. If $\left(A_{1}\right)$ is satisfied and $\left|\lambda^{\prime}\right|$ is a convex function on $\left[y_{1}, y_{2}\right]$, then

$$
\begin{aligned}
& \mid \frac{\lambda\left(y_{1}+y_{2}-\ell_{1}\right)+\lambda\left(y_{1}+y_{2}-\ell_{2}\right)}{2}-\frac{2^{\alpha-1} \Gamma(\alpha+1)}{\left(\ell_{2}-\ell_{1}\right)^{\alpha}} \\
& \quad \times\left\{\left(I_{\psi^{-1}}^{\alpha: \psi}\left(y_{1}+y_{2}-\ell_{2}\right)^{+}\right)\left[\lambda \circ \psi\left(\psi^{-1}\left(y_{1}+y_{2}-\frac{\ell_{1}+\ell_{2}}{2}\right)\right)\right]\right. \\
& \left.\quad+\left(I_{\psi^{-1}}^{\alpha: \psi}\left(y_{1}+y_{2}-\ell_{1}\right)^{-}\right)\left[\lambda \circ \psi\left(\psi^{-1}\left(y_{1}+y_{2}-\frac{\ell_{1}+\ell_{2}}{2}\right)\right)\right]\right\} \mid \\
& \leq \frac{\left(\ell_{2}-\ell_{1}\right)}{2(\alpha+1)}\left\{\left|\lambda^{\prime}\left(y_{1}\right)\right|+\left|\lambda^{\prime}\left(y_{2}\right)\right|-\left(\frac{\left|\lambda^{\prime}\left(\ell_{1}\right)\right|+\left|\lambda^{\prime}\left(\ell_{2}\right)\right|}{2}\right)\right\},
\end{aligned}
$$

for all $\ell_{1}, \ell_{2} \in\left[y_{1}, y_{2}\right]$.

Proof. By using Lemma 2, properties of modulus, and Jensen-Mercer inequality, we have

$$
\begin{aligned}
& \mid \frac{\lambda\left(y_{1}+y_{2}-\ell_{1}\right)+\lambda\left(y_{1}+y_{2}-\ell_{2}\right)}{2}-\frac{2^{\alpha-1} \Gamma(\alpha+1)}{\left(\ell_{2}-\ell_{1}\right)^{\alpha}} \\
& \times\left\{\left(I_{\psi^{-1}\left(y_{1}+y_{2}-\ell_{2}\right)^{+}}^{\alpha: \psi}\right)\left[\lambda \circ \psi\left(\psi^{-1}\left(y_{1}+y_{2}-\frac{\ell_{1}+\ell_{2}}{2}\right)\right)\right]\right. \\
& +\left(I_{\psi^{-1}\left(y_{1}+y_{2}-\ell_{1}\right)^{-}}^{\alpha: \psi}\left[\lambda \circ \psi\left(\psi^{-1}\left(y_{1}+y_{2}-\frac{\ell_{1}+\ell_{2}}{2}\right)\right)\right]\right\} \mid \\
& \leq \frac{\left(\ell_{2}-\ell_{1}\right)}{4}\left[\int_{0}^{1} \zeta^{\alpha}\left|\lambda^{\prime}\left(y_{1}+y_{2}-\left(\frac{1+\zeta}{2} \ell_{1}+\frac{1-\zeta}{2} \ell_{2}\right)\right)\right| \mathrm{d} \zeta\right. \\
& \left.+\int_{0}^{1} \zeta^{\alpha}\left|\lambda^{\prime}\left(y_{1}+y_{2}-\left(\frac{1-\zeta}{2} \ell_{1}+\frac{1+\zeta}{2} \ell_{2}\right)\right)\right| \mathrm{d} \zeta\right] \\
& \leq \frac{\left(\ell_{2}-\ell_{1}\right)}{4}\left[\int_{0}^{1} \zeta^{\alpha}\left\{\left|\lambda^{\prime}\left(y_{1}\right)\right|+\left|\lambda^{\prime}\left(y_{2}\right)\right|-\left(\frac{(1+\zeta)}{2}\left|\lambda^{\prime}\left(\ell_{1}\right)\right|+\frac{(1-\zeta)}{2}\left|\lambda^{\prime}\left(\ell_{2}\right)\right|\right)\right\} \mathrm{d} \zeta\right. \\
& \left.+\int_{0}^{1} \zeta^{\alpha}\left\{\left|\lambda^{\prime}\left(y_{1}\right)\right|+\left|\lambda^{\prime}\left(y_{2}\right)\right|-\left(\frac{(1-\zeta)}{2}\left|\lambda^{\prime}\left(\ell_{1}\right)\right|+\frac{(1+\zeta)}{2}\left|\lambda^{\prime}\left(\ell_{2}\right)\right|\right)\right\} \mathrm{d} \zeta\right]
\end{aligned}
$$

and after integration, we get required result. 
Remark 12. For $\psi(\gamma)=\gamma$ in Theorem 7 , we will get Theorem 4 proved in [29].

Lemma 3. If $\left(A_{1}\right)$ is satisfied and $\lambda:\left[y_{1}, y_{2}\right] \longrightarrow \mathfrak{R}$ is a differentiable function on $L_{1}\left[y_{1}, y_{2}\right]$, then

$$
\begin{aligned}
& \lambda\left(y_{1}+y_{2}-\frac{\ell_{1}+\ell_{2}}{2}\right)-\frac{2^{\alpha-1} \Gamma(\alpha+1)}{\left(\ell_{2}-\ell_{1}\right)^{\alpha}} \\
& \quad \times\left\{\left(I_{\psi^{-1}\left(y_{1}+y_{2}-\left(\left(\ell_{1}+\ell_{2}\right) / 2\right)\right)^{+}}^{\alpha: \psi}(\lambda \circ \psi)\left(\psi^{-1}\left(y_{1}+y_{2}-\ell_{1}\right)\right)\right.\right. \\
& +\left(I_{\psi^{-1}\left(y_{1}+y_{2}-\left(\left(\ell_{1}+\ell_{2}\right) / 2\right)\right)^{-}}^{\alpha: \psi}(\lambda \circ \psi)\left(\psi^{-1}\left(y_{1}+y_{2}-\ell_{2}\right)\right)\right\} \\
& =\frac{\left(\ell_{2}-\ell_{1}\right)}{4}\left[\int_{0}^{1} \zeta^{\alpha} \lambda^{\prime}\left(y_{1}+y_{2}-\left(\frac{\zeta}{2} \ell_{1}+\frac{2-\zeta}{2} \ell_{2}\right)\right) \mathrm{d} \zeta\right. \\
& \left.-\int_{0}^{1} \zeta^{\alpha} \lambda^{\prime}\left(y_{1}+y_{2}-\left(\frac{2-\zeta}{2} \ell_{1}+\frac{\zeta}{2} \ell_{2}\right)\right) \mathrm{d} \zeta\right]
\end{aligned}
$$

Proof. See the proof of Lemma 2.
Remark 14. For $\psi(\gamma)=\gamma$ in Lemma 3, we will get Lemma 2 proved in [27].

Theorem 8. If $\left(A_{1}\right)$ is satisfied and $\left|\lambda^{\prime}\right|$ is a convex function on $\left[y_{1}, y_{2}\right]$, then

$$
\begin{aligned}
& \mid \lambda\left(y_{1}+y_{2}-\frac{\ell_{1}+\ell_{2}}{2}\right)-\frac{2^{\alpha-1} \Gamma(\alpha+1)}{\left(\ell_{2}-\ell_{1}\right)^{\alpha}} \\
& \quad \times\left\{\left(I_{\psi^{-1}\left(y_{1}+y_{2}-\left(\left(\ell_{1}+\ell_{2}\right) / 2\right)\right)^{+}}^{\alpha: \psi}(\lambda \circ \psi)\left(\psi^{-1}\left(y_{1}+y_{2}-\ell_{1}\right)\right)\right.\right. \\
& \left.\quad+\left(I_{\psi^{-1}\left(y_{1}+y_{2}-\left(\left(\ell_{1}+\ell_{2}\right) / 2\right)\right)^{-}}^{\alpha: \psi}\right)(\lambda \circ \psi)\left(\psi^{-1}\left(y_{1}+y_{2}-\ell_{2}\right)\right)\right\} \mid \\
& \leq \frac{\left(\ell_{2}-\ell_{1}\right)}{2(\alpha+1)}\left\{\left|\lambda^{\prime}\left(y_{1}\right)\right|+\left|\lambda^{\prime}\left(y_{2}\right)\right|-\left(\frac{\left|\lambda^{\prime}\left(\ell_{1}\right)\right|+\left|\lambda^{\prime}\left(\ell_{2}\right)\right|}{2}\right)\right\},
\end{aligned}
$$

for all $\ell_{1}, \ell_{2} \in\left[y_{1}, y_{2}\right]$.

Proof. By using Lemma 3, properties of modulus, and Jensen-Mercer inequality, we have

Remark 13. For $\psi(\gamma)=\gamma, \ell_{1}=y_{1}$, and $\ell_{2}=y_{1}$ in Lemma 3, we will get Lemma 3 proved in [31].

$$
\begin{aligned}
& \mid \lambda\left(y_{1}+y_{2}-\frac{\ell_{1}+\ell_{2}}{2}\right)-\frac{2^{\alpha-1} \Gamma(\alpha+1)}{\left(\ell_{2}-\ell_{1}\right)^{\alpha}} \\
& \times\left\{\left(I_{\psi^{-1}\left(y_{1}+y_{2}-\left(\left(\ell_{1}+\ell_{2}\right) / 2\right)\right)^{+}}^{\alpha: \psi}(\lambda \circ \psi)\left(\psi^{-1}\left(y_{1}+y_{2}-\ell_{1}\right)\right)\right.\right. \\
& +\left(I_{\psi^{-1}\left(y_{1}+y_{2}-\left(\left(\ell_{1}+\ell_{2}\right) / 2\right)\right)^{-}}^{\alpha: \psi}(\lambda \circ \psi)\left(\psi^{-1}\left(y_{1}+y_{2}-\ell_{2}\right)\right)\right\} \mid \\
& \leq \frac{\left(\ell_{2}-\ell_{1}\right)}{4}\left[\int_{0}^{1} \zeta^{\alpha}\left|\lambda^{\prime}\left(y_{1}+y_{2}-\left(\frac{\zeta}{2} \ell_{1}+\frac{2-\zeta}{2} \ell_{2}\right)\right)\right| \mathrm{d} \zeta\right. \\
& \left.+\int_{0}^{1} \zeta^{\alpha}\left|\lambda^{\prime}\left(y_{1}+y_{2}-\left(\frac{2-\zeta}{2} \ell_{1}+\frac{\zeta}{2} \ell_{2}\right)\right)\right| \mathrm{d} \zeta\right] \\
& \leq \frac{\left(\ell_{2}-\ell_{1}\right)}{4}\left[\int_{0}^{1} \zeta^{\alpha}\left\{\left|\lambda^{\prime}\left(y_{1}\right)\right|+\left|\lambda^{\prime}\left(y_{2}\right)\right|-\left(\frac{\zeta}{2}\left|\lambda^{\prime}\left(\ell_{1}\right)\right|+\frac{(2-\zeta)}{2}\left|\lambda^{\prime}\left(\ell_{2}\right)\right|\right)\right\} \mathrm{d} \zeta\right. \\
& \left.+\int_{0}^{1} \zeta^{\alpha}\left\{\left|\lambda^{\prime}\left(y_{1}\right)\right|+\left|\lambda^{\prime}\left(y_{2}\right)\right|-\left(\frac{(2-\zeta)}{2}\left|\lambda^{\prime}\left(\ell_{1}\right)\right|+\frac{\zeta}{2}\left|\lambda^{\prime}\left(\ell_{2}\right)\right|\right)\right\} \mathrm{d} \zeta\right]
\end{aligned}
$$

After integration, we get required result. 
Remark 15. Taking $\psi(\gamma)=\gamma$ in Theorem 8, we will get Theorem 5 proved in [27].
Theorem 9. If $\left(A_{1}\right)$ is satisfied and $\left|\lambda^{\prime}\right|^{q}$ is convex function, then

$$
\begin{aligned}
& \mid \lambda\left(y_{1}+y_{2}-\frac{\ell_{1}+\ell_{2}}{2}\right)-\frac{2^{\alpha-1} \Gamma(\alpha+1)}{\left(\ell_{2}-\ell_{1}\right)^{\alpha}} \\
& \quad \times\left\{\left(I_{\psi^{-1}\left(y_{1}+y_{2}-\left(\left(\ell_{1}+\ell_{2}\right) / 2\right)\right)^{+}}^{\alpha: \psi}\right)(\lambda \circ \psi)\left(\psi^{-1}\left(y_{1}+y_{2}-\ell_{1}\right)\right)\right. \\
& \left.\quad+\left(I_{\psi^{-1}}^{\alpha: \psi}\left(y_{1}+y_{2}-\left(\left(\ell_{1}+\ell_{2}\right) / 2\right)\right)^{-}\right)(\lambda \circ \psi)\left(\psi^{-1}\left(y_{1}+y_{2}-\ell_{2}\right)\right)\right\} \mid \\
& \leq \frac{\left(\ell_{2}-\ell_{1}\right)}{4}\left(\frac{1}{p \alpha+1}\right)^{1 / p}\left[\left(\left|\lambda^{\prime}\left(y_{1}\right)\right|^{q}+\left|\lambda^{\prime}\left(y_{2}\right)\right|^{q}-\left(\frac{1}{4}\left|\lambda^{\prime}\left(\ell_{1}\right)\right|^{q}+\frac{3}{4}\left|\lambda^{\prime}\left(\ell_{2}\right)\right|^{q}\right)\right)^{1 / q}\right. \\
& \left.\quad+\left(\left|\lambda^{\prime}\left(y_{1}\right)\right|^{q}+\left|\lambda^{\prime}\left(y_{2}\right)\right|^{q}-\left(\frac{3}{4}\left|\lambda^{\prime}\left(\ell_{1}\right)\right|^{q}+\frac{1}{4}\left|\lambda^{\prime}\left(\ell_{2}\right)\right|^{q}\right)\right)^{1 / q}\right],
\end{aligned}
$$

where $q>1$ and $(1 / p)+(1 / q)=1$ for all $\ell_{1}, \ell_{2} \in\left[y_{1}, y_{2}\right]$.

Proof. Applying Lemma 3, Hölder and Jensen-Mercer inequalities, the fact that $\left|\lambda^{\prime}\right|^{q}$ is convex function, and properties of modulus, we have

$$
\begin{aligned}
& \mid \lambda\left(y_{1}+y_{2}-\frac{\ell_{1}+\ell_{2}}{2}\right)-\frac{2^{\alpha-1} \Gamma(\alpha+1)}{\left(\ell_{2}-\ell_{1}\right)^{\alpha}} \\
& \quad \times\left\{\left(I_{\psi^{-1}\left(y_{1}+y_{2}-\left(\left(\ell_{1}+\ell_{2}\right) / 2\right)\right)^{+}}^{\alpha: \psi}(\lambda \circ \psi)\left(\psi^{-1}\left(y_{1}+y_{2}-\ell_{1}\right)\right)\right.\right. \\
& \quad+\left(I_{\psi^{-1}\left(y_{1}+y_{2}-\left(\left(\ell_{1}+\ell_{2}\right) / 2\right)\right)^{-}}^{\alpha: \psi}\left(\lambda^{\circ} \psi\right)\left(\psi^{-1}\left(y_{1}+y_{2}-\ell_{2}\right)\right)\right\} \mid \\
& \leq \frac{\left(\ell_{2}-\ell_{1}\right)}{4} \int_{0}^{1} \zeta^{\alpha}\left|\lambda^{\prime}\left(y_{1}+y_{2}-\left(\frac{\zeta}{2} \ell_{1}+\frac{(2-\zeta)}{2} \ell_{2}\right)\right)\right| \mathrm{d} \zeta \\
& \quad+\frac{\left(\ell_{2}-\ell_{1}\right)}{4} \int_{0}^{1} \zeta^{\alpha}\left|\lambda^{\prime}\left(y_{1}+y_{2}-\left(\frac{(2-\zeta)}{2} \ell_{1}+\frac{\zeta}{2} \ell_{2}\right)\right)\right| \mathrm{d} \zeta \\
& \leq \frac{\left(\ell_{2}-\ell_{1}\right)}{4}\left(\int_{0}^{1} \zeta^{p \alpha} \mathrm{d} \zeta\right)^{1 / p}\left(\int_{0}^{1}\left|\lambda^{\prime}\left(y_{1}+y_{2}-\left(\frac{\zeta}{2} \ell_{1}+\frac{(2-\zeta)}{2} \ell_{2}\right)\right)\right|^{q} \mathrm{~d} \zeta\right)^{1 / q} \\
& \quad+\frac{\left(\ell_{2}-\ell_{1}\right)}{4}\left(\int_{0}^{1} \zeta^{p \alpha} \mathrm{d} \zeta\right)^{1 / p}\left(\int_{0}^{1}\left|\lambda^{\prime}\left(y_{1}+y_{2}-\left(\frac{(2-\zeta)}{2} \ell_{1}+\frac{\zeta}{2} \lambda^{\prime} \ell_{2}\right)\right)\right|^{q} \mathrm{~d} \zeta\right)^{1 / q} \\
& \leq \\
& \quad \frac{\left(\ell_{2}-\ell_{1}\right)}{4}\left(\frac{1}{p \alpha+1}\right)^{1 / p}\left(\left|\lambda^{\prime}\left(y_{1}\right)\right|^{q}+\left|\lambda^{\prime}\left(y_{2}\right)\right|^{q}-\left(\frac{1}{4}\left|\lambda^{\prime}\left(\ell_{1}\right)\right|^{q}+\frac{3}{4}\left|\lambda^{\prime}\left(\ell_{2}\right)\right|^{q}\right)\right)^{1 / q} \\
& \quad+\frac{\left(\ell_{2}-\ell_{1}\right)}{4}\left(\frac{1}{p \alpha+1}\right)^{1 / p}\left(\left|\lambda^{\prime}\left(y_{1}\right)\right|^{q}+\left|\lambda^{\prime}\left(y_{2}\right)\right|^{q}-\left(\frac{3}{4}\left|\lambda^{\prime}\left(\ell_{1}\right)\right|^{q}+\frac{1}{4}\left|\lambda^{\prime}\left(\ell_{2}\right)\right|^{q}\right)\right)^{1 / q} .
\end{aligned}
$$

After further simplifications, we get required result. 
Remark 16. For $\psi(\gamma)=\gamma$ in Theorem 9, we will get Theorem 6 proved in [27].
Lemma 4. If $\left(A_{1}\right)$ is satisfied and $\lambda:\left[y_{1}, y_{2}\right] \longrightarrow \mathfrak{R}$ is a twice differentiable function on $L_{1}\left[y_{1}, y_{2}\right]$, then

$$
\begin{aligned}
& \frac{2^{\alpha-1} \Gamma(\alpha+1)}{\left(\ell_{2}-\ell_{1}\right)^{\alpha}}\left\{\left(I_{\psi^{-1}\left(y_{1}+y_{2}-\left(\left(\ell_{1}+\ell_{2}\right) / 2\right)\right)^{+}}^{\alpha: \psi}\right)\left(\lambda \circ \psi\left(\psi^{-1}\left(y_{1}+y_{2}-\ell_{1}\right)\right)\right)\right. \\
& \left.\quad+\left(I_{\psi^{-1}\left(y_{1}+y_{2}-\left(\left(\ell_{1}+\ell_{2}\right) / 2\right)\right)^{-}}^{\alpha: \psi}\right)\left(\lambda \circ \psi\left(\psi^{-1}\left(y_{1}+y_{2}-\ell_{2}\right)\right)\right)\right\}-\lambda\left(y_{1}+y_{2}-\frac{\ell_{1}+\ell_{2}}{2}\right) \\
& =\frac{\left(\ell_{2}-\ell_{1}\right)^{2}}{8(\alpha+1)}\left[\int_{0}^{1}(1-\zeta)^{\alpha+1} \lambda^{\prime \prime}\left(y_{1}+y_{2}-\left(\frac{1+\zeta}{2} \ell_{1}+\frac{1-\zeta}{2} \ell_{2}\right)\right) \mathrm{d} \zeta\right. \\
& \left.\quad+\int_{0}^{1}(1-\zeta)^{\alpha+1} \lambda^{\prime \prime}\left(y_{1}+y_{2}-\left(\frac{1-\zeta}{2} \ell_{1}+\frac{1+\zeta}{2} \ell_{2}\right)\right) \mathrm{d} \zeta\right]
\end{aligned}
$$

Proof. It suffices to note that

where

$$
\begin{aligned}
& I=\frac{\left(\ell_{2}-\ell_{1}\right)^{2}}{8(\alpha+1)}\left\{I_{1}+I_{2}\right\} \\
& I_{1}= \int_{0}^{1}(1-\zeta)^{\alpha+1} \lambda^{\prime \prime}\left(y_{1}+y_{2}-\left(\frac{1+\zeta}{2} \ell_{1}+\frac{1-\zeta}{2} \ell_{2}\right)\right) \mathrm{d} \zeta \\
&=-\frac{2}{\left(\ell_{2}-\ell_{1}\right)} \lambda^{\prime}\left(y_{1}+y_{2}-\frac{\ell_{1}+\ell_{2}}{2}\right) \\
&+\frac{2(\alpha+1)}{\ell_{2}-\ell_{1}} \int_{0}^{1}(1-\zeta)^{\alpha} \lambda^{\prime}\left(y_{1}+y_{2}-\left(\frac{1+\zeta}{2} \ell_{1}+\frac{1-\zeta}{2} \ell_{2}\right)\right) \mathrm{d} \zeta \\
&=-\frac{2}{\left(\ell_{2}-\ell_{1}\right)} \lambda^{\prime}\left(y_{1}+y_{2}-\frac{\ell_{1}+\ell_{2}}{2}\right)-\frac{4(\alpha+1)}{\left(\ell_{2}-\ell_{1}\right)^{2}} \lambda\left(y_{1}+y_{2}-\frac{\ell_{1}+\ell_{2}}{2}\right) \\
&+\frac{4 \alpha(\alpha+1)}{\left(\ell_{2}-\ell_{1}\right)^{2}} \int_{0}^{1}(1-\zeta)^{\alpha-1} \lambda\left(y_{1}+y_{2}-\left(\frac{1+\zeta}{2} \ell_{1}+\frac{1-\zeta}{2} \ell_{2}\right)\right) \mathrm{d} \zeta \\
&=-\frac{2}{\left(\ell_{2}-\ell_{1}\right)} \lambda^{\prime}\left(y_{1}+y_{2}-\frac{\ell_{1}+\ell_{2}}{2}\right)-\frac{4(\alpha+1)}{\left(\ell_{2}-\ell_{1}\right)^{2}} \lambda\left(y_{1}+y_{2}-\frac{\ell_{1}+\ell_{2}}{2}\right) \\
&+\frac{2^{\alpha+2} \Gamma(\alpha+2)}{\left(\ell_{2}-\ell_{1}\right)^{\alpha+2}}\left(I_{\psi^{-1}}^{\alpha: \psi}\left(y_{1}+y_{2}-\left(\left(\ell_{1}+\ell_{2}\right) / 2\right)\right)^{+}\right)\left[\lambda \circ \psi\left(\psi^{-1}\left(y_{1}+y_{2}-\ell_{1}\right)\right)\right] \\
& I_{2}= \int_{0}^{1}(1-\zeta)^{\alpha+1} \lambda^{\prime \prime}\left(y_{1}+y_{2}-\left(\frac{1-\zeta}{2} \ell_{1}+\frac{1+\zeta}{2} \ell_{2}\right)\right) \mathrm{d} \zeta \\
&= \frac{2}{\left(\ell_{2}-\ell_{1}\right)} \lambda^{\prime}\left(y_{1}+y_{2}-\frac{\ell_{1}+\ell_{2}}{2}\right) \\
&-\frac{2(\alpha+1)}{\ell_{2}-\ell_{1}} \int_{0}^{1}(1-\zeta)^{\alpha} \lambda^{\prime}\left(y_{1}+y_{2}-\left(\frac{1-\zeta}{2} \ell_{1}+\frac{1+\zeta}{2} \ell_{2}\right)\right) \mathrm{d} \zeta \\
& \\
&
\end{aligned}
$$




$$
\begin{aligned}
= & \frac{2}{\left(\ell_{2}-\ell_{1}\right)} \lambda^{\prime}\left(y_{1}+y_{2}-\frac{\ell_{1}+\ell_{2}}{2}\right)-\frac{4(\alpha+1)}{\left(\ell_{2}-\ell_{1}\right)^{2}} \lambda\left(y_{1}+y_{2}-\frac{\ell_{1}+\ell_{2}}{2}\right) \\
& +\frac{4 \alpha(\alpha+1)}{\left(\ell_{2}-\ell_{1}\right)^{2}} \int_{0}^{1}(1-\zeta)^{\alpha-1} \lambda\left(y_{1}+y_{2}-\left(\frac{1-\zeta}{2} \ell_{1}+\frac{1+\zeta}{2} \ell_{2}\right)\right) \mathrm{d} \zeta \\
= & \frac{2}{\left(\ell_{2}-\ell_{1}\right)} \lambda^{\prime}\left(y_{1}+y_{2}-\frac{\ell_{1}+\ell_{2}}{2}\right)-\frac{4(\alpha+1)}{\left(\ell_{2}-\ell_{1}\right)^{2}} \lambda\left(y_{1}+y_{2}-\frac{\ell_{1}+\ell_{2}}{2}\right) \\
& +\frac{2^{\alpha+2} \Gamma(\alpha+2)}{\left(\ell_{2}-\ell_{1}\right)^{\alpha+2}}\left(I_{\psi^{-1}}^{\alpha: \psi}\left(y_{1}+y_{2}-\left(\left(\ell_{1}+\ell_{2}\right) / 2\right)\right)^{-}\right)\left[\lambda \circ \psi\left(\psi^{-1}\left(y_{1}+y_{2}-\ell_{2}\right)\right)\right] .
\end{aligned}
$$

Substituting (73) and (74) in (72), we get (71).

Corollary 1. If we set $\ell_{1}=y_{1}$ and $\ell_{2}=y_{2}$, we get

$$
\begin{aligned}
& \frac{2^{\alpha-1} \Gamma(\alpha+1)}{\left(y_{2}-y_{1}\right)^{\alpha}}\left\{\left(I_{\psi^{-1}}^{\alpha: \psi}\left(\left(y_{1}+y_{2}\right) / 2\right)^{+}\right)\left(\lambda \circ \psi\left(\psi^{-1}\left(y_{2}\right)\right)\right)\right. \\
& \left.+\left(I_{\psi^{-1}}^{\alpha: \psi}\left(\left(y_{1}+y_{2}\right) / 2\right)^{-}\right)\left(\lambda \circ \psi\left(\psi^{-1}\left(y_{1}\right)\right)\right)\right\}-\lambda\left(\frac{y_{1}+y_{2}}{2}\right) \\
& =\frac{\left(y_{2}-y_{1}\right)^{2}}{8(\alpha+1)}\left[\int_{0}^{1}(1-\zeta)^{\alpha+1} \lambda^{\prime \prime}\left(\frac{1+\zeta}{2} y_{1}+\frac{1-\zeta}{2} y_{2}\right) \mathrm{d} \zeta\right. \\
& \left.\quad+\int_{0}^{1}(1-\zeta)^{\alpha+1} \lambda^{\prime \prime}\left(\frac{1-\zeta}{2} y_{1}+\frac{1+\zeta}{2} y_{2}\right) \mathrm{d} \zeta\right]
\end{aligned}
$$

Remark 17. If we set $\psi(\gamma)=\gamma$ in Lemma 4 , we get Lemma 2 of [29].

Moreover, if we set $\ell_{1}=y_{1}$ and $\ell_{2}=y_{2}$, we obtain Lemma 1 of [32].

Remark 18. For $\psi(\gamma)=\gamma, \alpha=1, \ell_{1}=y_{1}$, and $\ell_{2}=y_{2}$ in Lemma 4 , it reduces to Lemma 2 proved in [32].

Theorem 10. If $\left(A_{1}\right)$ is satisfied and $\left|\lambda^{\prime \prime}\right|$ is a convex function on $\left[y_{1}, y_{2}\right]$, then

$$
\begin{aligned}
& \mid \frac{2^{\alpha-1} \Gamma(\alpha+1)}{\left(\ell_{2}-\ell_{1}\right)^{\alpha}}\left\{\left(I_{\psi^{-1}\left(y_{1}+y_{2}-\left(\left(\ell_{1}+\ell_{2}\right) / 2\right)\right)^{+}}^{\alpha: \psi}\left(\lambda \circ \psi\left(\psi^{-1}\left(y_{1}+y_{2}-\ell_{1}\right)\right)\right)\right.\right. \\
& \quad+\left(I_{\psi^{-1}\left(y_{1}+y_{2}-\left(\left(\ell_{1}+\ell_{2}\right) / 2\right)\right)^{-}}^{\alpha: \psi}\left(\lambda \circ \psi\left(\psi^{-1}\left(y_{1}+y_{2}-\ell_{2}\right)\right)\right)\right\}-\lambda\left(y_{1}+y_{2}-\frac{\ell_{1}+\ell_{2}}{2}\right) \mid \\
& \leq \frac{\left(\ell_{2}-\ell_{1}\right)^{2}}{4(\alpha+1)(\alpha+2)}\left\{\left|\lambda^{\prime \prime}\left(y_{1}\right)\right|+\left|\lambda^{\prime \prime}\left(y_{2}\right)\right|-\left(\frac{\left|\lambda^{\prime \prime}\left(\ell_{1}\right)\right|+\left|\lambda^{\prime \prime}\left(\ell_{2}\right)\right|}{2}\right)\right\} .
\end{aligned}
$$

Proof. By using Lemma 4, properties of modulus, and Jensen-Mercer inequality, we have 


$$
\begin{aligned}
& \mid \frac{2^{\alpha-1} \Gamma(\alpha+1)}{\left(\ell_{2}-\ell_{1}\right)^{\alpha}}\left\{\left(I_{\left.\psi^{-1}\left(y_{1}+y_{2}-\left(\left(\ell_{1}+\ell_{2}\right) / 2\right)\right)^{+}\right)\left(\lambda \circ \psi\left(\psi^{-1}\left(y_{1}+y_{2}-\ell_{1}\right)\right)\right)}\right.\right. \\
& \left.\quad+\left(I_{\psi^{-1}}^{\alpha: \psi}\left(y_{1}+y_{2}-\left(\left(\ell_{1}+\ell_{2}\right) / 2\right)\right)^{-}\right)\left(\lambda \circ \psi\left(\psi^{-1}\left(y_{1}+y_{2}-\ell_{2}\right)\right)\right)\right\}-\lambda\left(y_{1}+y_{2}-\frac{\ell_{1}+\ell_{2}}{2}\right) \mid \\
& \leq \frac{\left(\ell_{2}-\ell_{1}\right)^{2}}{8(\alpha+1)}\left[\int_{0}^{1}(1-\zeta)^{\alpha+1}\left|\lambda^{\prime \prime}\left(y_{1}+y_{2}-\left(\frac{1+\zeta}{2} \ell_{1}+\frac{1-\zeta}{2} \ell_{2}\right)\right)\right| \mathrm{d} \zeta\right. \\
& \left.\quad+\int_{0}^{1}(1-\zeta)^{\alpha+1}\left|\lambda^{\prime \prime}\left(y_{1}+y_{2}-\left(\frac{1-\zeta}{2} \ell_{1}+\frac{1+\zeta}{2} \ell_{2}\right)\right)\right| \mathrm{d} \zeta\right] \\
& \leq \frac{\left(\ell_{2}-\ell_{1}\right)^{2}}{8(\alpha+1)}\left[\int_{0}^{1}(1-\zeta)^{\alpha+1}\left\{\left|\lambda^{\prime \prime}\left(y_{1}\right)\right|+\left|\lambda^{\prime \prime}\left(y_{2}\right)\right|-\left(\frac{1+\zeta}{2}\left|\lambda^{\prime \prime}\left(\ell_{1}\right)\right|+\frac{1-\zeta}{2}\left|\lambda^{\prime \prime}\left(\ell_{2}\right)\right|\right)\right\} \mathrm{d} \zeta\right. \\
& \left.\quad+\int_{0}^{1}(1-\zeta)^{\alpha+1}\left\{\left|\lambda^{\prime \prime}\left(y_{1}\right)\right|+\left|\lambda^{\prime \prime}\left(y_{2}\right)\right|-\left(\frac{1-\zeta}{2}\left|\lambda^{\prime \prime}\left(\ell_{1}\right)\right|+\frac{1+\zeta}{2}\left|\lambda^{\prime \prime}\left(\ell_{2}\right)\right|\right)\right\} \mathrm{d} \zeta\right],
\end{aligned}
$$

and after integration, we get required result.

Corollary 2. If we set $\ell_{1}=y_{1}$ and $\ell_{2}=y_{2}$ in Theorem 10, we get

$$
\begin{aligned}
& \mid \frac{2^{\alpha-1} \Gamma(\alpha+1)}{\left(y_{2}-y_{1}\right)^{\alpha}}\left\{\left(I_{\psi^{-1}}^{\alpha: \psi}\left(\left(y_{1}+y_{2}\right) / 2\right)^{+}\right)\left(\lambda \circ \psi\left(\psi^{-1}\left(y_{2}\right)\right)\right)\right. \\
& \left.\quad+\left(I_{\psi^{-1}}^{\alpha: \psi}\left(\left(y_{1}+y_{2}\right) / 2\right)^{-}\right)\left(\lambda \circ \psi\left(\psi^{-1}\left(y_{1}\right)\right)\right)\right\}-\lambda\left(\frac{y_{1}+y_{2}}{2}\right) \mid \\
& \leq \frac{\left(y_{2}-y_{1}\right)^{2}}{4(\alpha+1)(\alpha+2)}\left\{\left(\frac{\left|\lambda^{\prime \prime}\left(y_{1}\right)\right|+\left|\lambda^{\prime \prime}\left(y_{2}\right)\right|}{2}\right)\right\} .
\end{aligned}
$$

Remark 19. If we set $\psi(\gamma)=\gamma$ in Theorem 10, we obtain Theorem 5 of [29].

Moreover, if we set $\ell_{1}=y_{1}$ and $\ell_{2}=y_{2}$, we get Theorem 5 of [32].

Corollary 3. If we set $\psi(\gamma)=\gamma, \ell_{1}=y_{1}, \ell_{2}=y_{2}$, and $\alpha=1$ in Theorem 10, we get Proposition 1 of [33]:

$$
\left|\frac{1}{y_{2}-y_{1}} \int_{y_{1}}^{y_{2}} \lambda(x) \mathrm{d} x-\lambda\left(\frac{y_{1}+y_{2}}{2}\right)\right| \leq \frac{\left(y_{2}-y_{1}\right)^{2}}{24}\left\{\left(\frac{\left|\lambda^{\prime \prime}\left(y_{1}\right)\right|+\left|\lambda^{\prime \prime}\left(y_{2}\right)\right|}{2}\right)\right\} .
$$

Theorem 11. If $\left(A_{1}\right)$ is satisfied and $\left|\lambda^{\prime \prime}\right|^{q}$ is convex function, then

$$
\begin{aligned}
& \mid \frac{2^{\alpha-1} \Gamma(\alpha+1)}{\left(\ell_{2}-\ell_{1}\right)^{\alpha}}\left\{\left(I_{\psi^{-1}\left(y_{1}+y_{2}-\left(\left(\ell_{1}+\ell_{2}\right) / 2\right)\right)^{+}}^{\alpha: \psi}\right)\left(\lambda \circ \psi\left(\psi^{-1}\left(y_{1}+y_{2}-\ell_{1}\right)\right)\right)\right. \\
& +\left(I_{\psi^{-1}\left(y_{1}+y_{2}-\left(\left(\ell_{1}+\ell_{2}\right) / 2\right)\right)^{-}}^{\alpha: \psi}\left(\lambda \circ \psi\left(\psi^{-1}\left(y_{1}+y_{2}-\ell_{2}\right)\right)\right)\right\}-\lambda\left(y_{1}+y_{2}-\frac{\ell_{1}+\ell_{2}}{2}\right) \mid \\
& \leq \frac{\left(\ell_{2}-\ell_{1}\right)^{2}}{8(\alpha+1)}\left(\frac{1}{p(\alpha+1)+1}\right)^{1 / p}\left[\left(\left|\lambda^{\prime \prime}\left(y_{1}\right)\right|^{q}+\left|\lambda^{\prime \prime}\left(y_{2}\right)\right|^{q}-\frac{\left|3 \lambda^{\prime \prime}\left(\ell_{1}\right)\right|^{q}+\left|\lambda^{\prime \prime}\left(\ell_{2}\right)\right|^{q}}{4}\right)^{1 / q}\right. \\
& \left.\left.+\left(\left|\lambda^{\prime \prime}\left(y_{1}\right)\right|^{q}+\left|\lambda^{\prime \prime}\left(y_{2}\right)\right|^{q}-\frac{\left|\lambda^{\prime \prime}\left(\ell_{1}\right)\right|^{q}+3\left|\lambda^{\prime \prime}\left(\ell_{2}\right)\right|^{q}}{4}\right)\right)^{1 / q}\right] \text {, }
\end{aligned}
$$


where $q>1$ and $(1 / p)+(1 / q)=1$ for all $\ell_{1}, \ell_{2} \in\left[y_{1}, y_{2}\right]$
Proof. From Lemma 4, Hölder and Jensen-Mercer inequalities, the fact that $\left|\lambda^{\prime \prime}\right|^{q}$ is convex function, and properties of modulus, we have

$$
\begin{aligned}
& \mid \frac{2^{\alpha-1} \Gamma(\alpha+1)}{\left(\ell_{2}-\ell_{1}\right)^{\alpha}}\left\{\left(\begin{array}{c}
I^{\alpha: \psi} \\
\psi^{-1}\left(y_{1}+y_{2}-\frac{\ell_{1}+\ell_{2}}{2}\right)^{+}
\end{array}\right)\left(\lambda \circ \psi\left(\psi^{-1}\left(y_{1}+y_{2}-\ell_{1}\right)\right)\right)\right. \\
& \left.+\left(I_{\psi^{-1}\left(y_{1}+y_{2}-\left(\left(\ell_{1}+\ell_{2}\right) / 2\right)\right)^{-}}^{\alpha: \psi}\right)\left(\lambda \circ \psi\left(\psi^{-1}\left(y_{1}+y_{2}-\ell_{2}\right)\right)\right)\right\}-\lambda\left(y_{1}+y_{2}-\frac{\ell_{1}+\ell_{2}}{2}\right) \mid \\
& \leq \frac{\left(\ell_{2}-\ell_{1}\right)^{2}}{8(\alpha+1)}\left[\int_{0}^{1}(1-\zeta)^{\alpha+1}\left|\lambda^{\prime \prime}\left(y_{1}+y_{2}-\left(\frac{1+\zeta}{2} \ell_{1}+\frac{1-\zeta}{2} \ell_{2}\right)\right)\right| \mathrm{d} \zeta\right. \\
& \left.+\int_{0}^{1}(1-\zeta)^{\alpha+1}\left|\lambda^{\prime \prime}\left(y_{1}+y_{2}-\left(\frac{1-\zeta}{2} \ell_{1}+\frac{1+\zeta}{2} \ell_{2}\right)\right)\right| \mathrm{d} \zeta\right] \\
& \leq \frac{\left(\ell_{2}-\ell_{1}\right)^{2}}{8(\alpha+1)}\left[\left(\int_{0}^{1}(1-\zeta)^{p(\alpha+1)} \mathrm{d} \zeta\right)^{1 / p}\left(\int_{0}^{1}\left|\lambda^{\prime \prime}\left(y_{1}+y_{2}-\left(\frac{1+\zeta}{2} \ell_{1}+\frac{1-\zeta}{2} \ell_{2}\right)\right)\right|^{q} \mathrm{~d} \zeta\right)^{1 / q}\right. \\
& \left.+\left(\int_{0}^{1}(1-\zeta)^{p(\alpha+1)} \mathrm{d} \zeta\right)^{1 / p}\left(\int_{0}^{1}\left|\lambda^{\prime \prime}\left(y_{1}+y_{2}-\left(\frac{1-\zeta}{2} \ell_{1}+\frac{1+\zeta}{2} \ell_{2}\right)\right)\right|^{q} \mathrm{~d} \zeta\right)^{1 / q}\right] \\
& \leq \frac{\left(\ell_{2}-\ell_{1}\right)^{2}}{8(\alpha+1)}\left(\int_{0}^{1}(1-\zeta)^{p(\alpha+1)} \mathrm{d} \zeta\right)^{1 / p} \\
& \times\left[\left(\int_{0}^{1}\left(\left|\lambda^{\prime \prime}\left(y_{1}\right)\right|^{q}+\left|\lambda^{\prime \prime}\left(y_{2}\right)\right|^{q}-\frac{1+\zeta}{2}\left|\lambda^{\prime \prime}\left(\ell_{1}\right)\right|^{q}-\frac{1-\zeta}{2}\left|\lambda^{\prime \prime}\left(\ell_{2}\right)\right|^{q}\right) \mathrm{d} \zeta\right)^{1 / q}\right. \\
& \left.+\left(\int_{0}^{1}\left(\left|\lambda^{\prime \prime}\left(y_{1}\right)\right|^{q}+\left|\lambda^{\prime \prime}\left(y_{2}\right)\right|^{q}-\frac{1-\zeta}{2}\left|\lambda^{\prime \prime}\left(\ell_{1}\right)\right|^{q}-\frac{1+\zeta}{2}\left|\lambda^{\prime \prime}\left(\ell_{2}\right)\right|^{q}\right) \mathrm{d} \zeta\right)^{1 / q}\right] \\
& =\frac{\left(\ell_{2}-\ell_{1}\right)^{2}}{8(\alpha+1)}\left(\frac{1}{p(\alpha+1)+1}\right)^{1 / p}\left[\left(\left|\lambda^{\prime \prime}\left(y_{1}\right)\right|^{q}+\left|\lambda^{\prime \prime}\left(y_{2}\right)\right|^{q}-\frac{\left|3 \lambda^{\prime \prime}\left(\ell_{1}\right)\right|^{q}+\left|\lambda^{\prime \prime}\left(\ell_{2}\right)\right|^{q}}{4}\right)^{1 / q}\right. \\
& \left.\left.+\left(\left.|| \lambda^{\prime \prime}\left(y_{1}\right)\right|^{q}+\left|\lambda^{\prime \prime}\left(y_{2}\right)\right|^{q}-\left.\frac{\left|\lambda^{\prime \prime}\left(\ell_{1}\right)\right|^{q}+3\left|\lambda^{\prime \prime}\left(\ell_{2}\right)\right|^{q}}{4}\right|^{q}\right)\right)^{1 / q}\right] .
\end{aligned}
$$

Remark 20. If we set $\psi(\gamma)=\gamma$ in Theorem 11, we get Theorem 6 of [29].
Lemma 5. If $\left(A_{1}\right)$ satisfied and $\lambda:\left[y_{1}, y_{2}\right] \longrightarrow \mathfrak{R}$ is a twice differentiable function on $L_{1}\left[y_{1}, y_{2}\right]$, then 


$$
\begin{aligned}
& \frac{2^{\alpha-1} \Gamma(\alpha+1)}{\left(\ell_{2}-\ell_{1}\right)^{\alpha}}\left\{\left(I_{\psi^{-1}\left(y_{1}+y_{2}-\left(\left(\ell_{1}+\ell_{2}\right) / 2\right)\right)^{+}}^{\alpha: \psi}\right)\left(\lambda \circ \psi\left(\psi^{-1}\left(y_{1}+y_{2}-\ell_{1}\right)\right)\right)\right. \\
& \left.\quad+\left(I_{\psi^{-1}\left(y_{1}+y_{2}-\left(\left(\ell_{1}+\ell_{2}\right) / 2\right)\right)^{-}}^{\alpha: \psi}\right)\left(\lambda \circ \psi\left(\psi^{-1}\left(y_{1}+y_{2}-\ell_{2}\right)\right)\right)\right\}-\lambda\left(y_{1}+y_{2}-\frac{\ell_{1}+\ell_{2}}{2}\right) \\
& =\frac{\left(\ell_{2}-\ell_{1}\right)^{2}}{8(\alpha+1)}\left[\int_{0}^{1} \zeta^{\alpha+1} \lambda^{\prime \prime}\left(y_{1}+y_{2}-\left(\frac{2-\zeta}{2} \ell_{1}+\frac{\zeta}{2} \ell_{2}\right)\right) \mathrm{d} \zeta\right. \\
& \left.\quad+\int_{0}^{1} \zeta^{\alpha+1} \lambda^{\prime \prime}\left(y_{1}+y_{2}-\left(\frac{\zeta_{2}}{2} \ell_{1}+\frac{2-\zeta}{2} \ell_{2}\right)\right) \mathrm{d} \zeta\right]
\end{aligned}
$$

Proof. It suffices to note that

$$
I=\frac{\left(\ell_{2}-\ell_{1}\right)^{2}}{8(\alpha+1)}\left\{I_{1}+I_{2}\right\},
$$

$$
\begin{aligned}
& I_{1}=\int_{0}^{1} \zeta^{\alpha+1} \lambda^{\prime \prime}\left(y_{1}+y_{2}-\left(\frac{2-\zeta}{2} \ell_{1}+\frac{\zeta}{2} \ell_{2}\right)\right) \mathrm{d} \zeta \\
& =-\frac{2}{\left(\ell_{2}-\ell_{1}\right)} \lambda^{\prime}\left(y_{1}+y_{2}-\frac{\ell_{1}+\ell_{2}}{2}\right) \\
& +\frac{2(\alpha+1)}{\ell_{2}-\ell_{1}} \int_{0}^{1} \zeta^{\alpha} \lambda^{\prime}\left(y_{1}+y_{2}-\left(\frac{2-\zeta}{2} \ell_{1}+\frac{\zeta}{2} \ell_{2}\right)\right) \mathrm{d} \zeta \\
& =-\frac{2}{\left(\ell_{2}-\ell_{1}\right)} \lambda^{\prime}\left(y_{1}+y_{2}-\frac{\ell_{1}+\ell_{2}}{2}\right)-\frac{4(\alpha+1)}{\left(\ell_{2}-\ell_{1}\right)^{2}} \lambda\left(y_{1}+y_{2}-\frac{\ell_{1}+\ell_{2}}{2}\right) \\
& +\frac{4 \alpha(\alpha+1)}{\left(\ell_{2}-\ell_{1}\right)^{2}} \int_{0}^{1} \zeta^{\alpha-1} \lambda\left(y_{1}+y_{2}-\left(\frac{2-\zeta}{2} \ell_{1}+\frac{\zeta}{2} \ell_{2}\right)\right) \mathrm{d} \zeta \\
& =-\frac{2}{\left(\ell_{2}-\ell_{1}\right)} \lambda^{\prime}\left(y_{1}+y_{2}-\frac{\ell_{1}+\ell_{2}}{2}\right)-\frac{4(\alpha+1)}{\left(\ell_{2}-\ell_{1}\right)^{2}} \lambda\left(y_{1}+y_{2}-\frac{\ell_{1}+\ell_{2}}{2}\right) \\
& +\frac{2^{\alpha+2} \Gamma(\alpha+2)}{\left(\ell_{2}-\ell_{1}\right)^{\alpha+2}}\left(I_{\psi^{-1}\left(y_{1}+y_{2}-\left(\left(\ell_{1}+\ell_{2}\right) / 2\right)\right)^{+}}^{\alpha: \psi}\left[\lambda \circ \psi\left(\psi^{-1}\left(y_{1}+y_{2}-\ell_{1}\right)\right)\right]\right. \text {, } \\
& I_{2}=\int_{0}^{1} \zeta^{\alpha+1} \lambda^{\prime \prime}\left(y_{1}+y_{2}-\left(\frac{\zeta}{2} \ell_{1}+\frac{2-\zeta}{2} \ell_{2}\right)\right) \mathrm{d} \zeta \\
& =\frac{2}{\left(\ell_{2}-\ell_{1}\right)} \lambda^{\prime}\left(y_{1}+y_{2}-\frac{\ell_{1}+\ell_{2}}{2}\right) \\
& -\frac{2(\alpha+1)}{\ell_{2}-\ell_{1}} \int_{0}^{1} \zeta^{\alpha} \lambda^{\prime}\left(y_{1}+y_{2}-\left(\frac{\zeta}{2} \ell_{1}+\frac{2-\zeta}{2} \ell_{2}\right)\right) \mathrm{d} \zeta \\
& =\frac{2}{\left(\ell_{2}-\ell_{1}\right)} \lambda^{\prime}\left(y_{1}+y_{2}-\frac{\ell_{1}+\ell_{2}}{2}\right)-\frac{4(\alpha+1)}{\left(\ell_{2}-\ell_{1}\right)^{2}} \lambda\left(y_{1}+y_{2}-\frac{\ell_{1}+\ell_{2}}{2}\right) \\
& +\frac{4 \alpha(\alpha+1)}{\left(\ell_{2}-\ell_{1}\right)^{2}} \int_{0}^{1} \zeta^{\alpha-1} \lambda\left(y_{1}+y_{2}-\left(\frac{\zeta}{2} \ell_{1}+\frac{2-\zeta}{2} \ell_{2}\right)\right) \mathrm{d} \zeta \\
& =\frac{2}{\left(\ell_{2}-\ell_{1}\right)} \lambda^{\prime}\left(y_{1}+y_{2}-\frac{\ell_{1}+\ell_{2}}{2}\right)-\frac{4(\alpha+1)}{\left(\ell_{2}-\ell_{1}\right)^{2}} \lambda\left(y_{1}+y_{2}-\frac{\ell_{1}+\ell_{2}}{2}\right) \\
& +\frac{2^{\alpha+2} \Gamma(\alpha+2)}{\left(\ell_{2}-\ell_{1}\right)^{\alpha+2}}\left(I_{\psi^{-1}\left(y_{1}+y_{2}-\left(\left(\ell_{1}+\ell_{2}\right) / 2\right)\right)^{-}}^{\alpha:}\right)\left[\lambda \circ \psi\left(\psi^{-1}\left(y_{1}+y_{2}-\ell_{2}\right)\right)\right] \text {. }
\end{aligned}
$$




$$
\begin{aligned}
& \frac{2^{\alpha-1} \Gamma(\alpha+1)}{\left(y_{2}-y_{1}\right)^{\alpha}}\left\{\left(I_{\psi^{-1}}^{\alpha: \psi}\left(\left(y_{1}+y_{2}\right) / 2\right)^{+}\right)\left(\lambda \circ \psi\left(\psi^{-1}\left(y_{2}\right)\right)\right)\right. \\
& \left.+\left(I_{\psi^{-1}}^{\alpha: \psi}\left(\left(y_{1}+y_{2}\right) / 2\right)^{-}\right)\left(\lambda \circ \psi\left(\psi^{-1}\left(y_{1}\right)\right)\right)\right\}-\lambda\left(\frac{y_{1}+y_{2}}{2}\right) \\
& =\frac{\left(y_{2}-y_{1}\right)^{2}}{8(\alpha+1)}\left[\int_{0}^{1} \zeta^{\alpha+1} \lambda^{\prime \prime}\left(\frac{2-\zeta}{2} y_{1}+\frac{\zeta}{2} y_{2}\right) \mathrm{d} \zeta+\int_{0}^{1} \zeta^{\alpha+1} \lambda^{\prime \prime}\left(\frac{\zeta}{2} y_{1}+\frac{2-\zeta}{2} y_{2}\right) \mathrm{d} \zeta\right] .
\end{aligned}
$$

Corollary 5. If we set $\psi(\gamma)=\gamma$, we get

$$
\begin{aligned}
& \frac{2^{\alpha-1} \Gamma(\alpha+1)}{\left(\ell_{2}-\ell_{1}\right)^{\alpha}}\left\{\left(I_{\left(y_{1}+y_{2}-\left(\left(\ell_{1}+\ell_{2}\right) / 2\right)\right)^{+}}^{\alpha}\right)\left(\lambda\left(y_{1}+y_{2}-\ell_{1}\right)\right)\right. \\
& \left.\quad+\left(I_{\left(y_{1}+y_{2}-\left(\left(\ell_{1}+\ell_{2}\right) / 2\right)\right)^{-}}^{\alpha}\right)\left(\lambda\left(y_{1}+y_{2}-\ell_{2}\right)\right)\right\}-\lambda\left(y_{1}+y_{2}-\frac{\ell_{1}+\ell_{2}}{2}\right) \\
& =\frac{\left(\ell_{2}-\ell_{1}\right)^{2}}{8(\alpha+1)}\left[\int_{0}^{1} \zeta^{\alpha+1} \lambda^{\prime \prime}\left(y_{1}+y_{2}-\left(\frac{2-\zeta}{2} \ell_{1}+\frac{\zeta}{2} \ell_{2}\right)\right) \mathrm{d} \zeta\right. \\
& \left.\quad+\int_{0}^{1} \zeta^{\alpha+1} \lambda^{\prime \prime}\left(y_{1}+y_{2}-\left(\frac{\zeta}{2} \ell_{1}+\frac{2-\zeta}{2} \ell_{2}\right)\right) \mathrm{d} \zeta\right]
\end{aligned}
$$

Moreover, if we set $\ell_{1}=y_{1}$ and $\ell_{2}=y_{2}$, we get

$$
\begin{aligned}
& \frac{2^{\alpha-1} \Gamma(\alpha+1)}{\left(y_{2}-y_{1}\right)^{\alpha}}\left\{I^{\alpha}\left(\left(y_{1}+y_{2}\right) / 2\right)^{+} \lambda\left(y_{2}\right)+I_{\left(\left(y_{1}+y_{2}\right) / 2\right)^{-}}^{\alpha}\left(y_{1}\right)\right\}-\lambda\left(\frac{y_{1}+y_{2}}{2}\right) \\
& =\frac{\left(y_{2}-y_{1}\right)^{2}}{8(\alpha+1)}\left[\int_{0}^{1} \zeta^{\alpha+1} \lambda^{\prime \prime}\left(\frac{2-\zeta}{2} y_{1}+\frac{\zeta}{2} y_{2}\right) \mathrm{d} \zeta+\int_{0}^{1} \zeta^{\alpha+1} \lambda^{\prime \prime}\left(\frac{\zeta}{2} y_{1}+\frac{2-\zeta}{2} y_{2}\right) \mathrm{d} \zeta\right] .
\end{aligned}
$$

Remark 21. By using Lemma 5, we can get the same results of Theorems 10 and 11, so we omit their proof here.
Lemma 6. If $\left(A_{1}\right)$ is satisfied and $\lambda:\left[y_{1}, y_{2}\right] \longrightarrow \mathfrak{R}$ is a twice differentiable function on $L_{1}\left[y_{1}, y_{2}\right]$, then

$$
\begin{aligned}
& \frac{2^{\alpha-2} \Gamma(\alpha)}{\left(\ell_{2}-\ell_{1}\right)^{\alpha-1}}\left\{\left(I_{\psi^{-1}\left(y_{1}+y_{2}-\left(\left(\ell_{1}+\ell_{2}\right) / 2\right)\right)^{+}}^{\alpha-1:}\right)\left(\lambda \circ \psi\left(\psi^{-1}\left(y_{1}+y_{2}-\ell_{1}\right)\right)\right)\right. \\
& \left.\quad+\left(I_{\psi^{-1}\left(y_{1}+y_{2}-\left(\left(\ell_{1}+\ell_{2}\right) / 2\right)\right)^{-}}^{\alpha-1:}\right)\left(\lambda \circ \psi\left(\psi^{-1}\left(y_{1}+y_{2}-\ell_{2}\right)\right)\right)\right\}-\lambda\left(y_{1}+y_{2}-\frac{\ell_{1}+\ell_{2}}{2}\right) \\
& =\frac{\left(\ell_{2}-\ell_{1}\right)^{2}}{\alpha \cdot 2^{2-\alpha}}\left[\int_{0}^{1 / 2} \zeta^{\alpha} \lambda^{\prime \prime}\left(y_{1}+y_{2}-\left(\zeta \ell_{2}+(1-\zeta) \ell_{1}\right)\right) \mathrm{d} \zeta\right. \\
& \left.\quad+\int_{1 / 2}^{1}(1-\zeta)^{\alpha} \lambda^{\prime \prime}\left(y_{1}+y_{2}-\left(\zeta \ell_{2}+(1-\zeta) \ell_{1}\right)\right) \mathrm{d} \zeta\right]
\end{aligned}
$$


Proof. It suffices to note that

$$
I=\frac{\left(\ell_{2}-\ell_{1}\right)^{2}}{\alpha \cdot 2^{1-\alpha}}\left\{I_{1}+I_{2}\right\},
$$

$$
\begin{aligned}
& I_{1}=\int_{0}^{1 / 2} \zeta^{\alpha} \lambda^{\prime \prime}\left(y_{1}+y_{2}-\left(\zeta \ell_{2}+(1-\zeta) \ell_{1}\right)\right) \mathrm{d} \zeta \\
& =-\frac{1}{2^{\alpha}\left(\ell_{2}-\ell_{1}\right)} \lambda^{\prime}\left(y_{1}+y_{2}-\frac{\ell_{1}+\ell_{2}}{2}\right) \\
& +\frac{\alpha}{\ell_{2}-\ell_{1}} \int_{0}^{1 / 2} \zeta^{\alpha-1} \lambda^{\prime}\left(y_{1}+y_{2}-\left((1-\zeta) \ell_{1}+\zeta \ell_{2}\right)\right) \mathrm{d} \zeta \\
& =-\frac{1}{2^{\alpha}\left(\ell_{2}-\ell_{1}\right)} \lambda^{\prime}\left(y_{1}+y_{2}-\frac{\ell_{1}+\ell_{2}}{2}\right)-\frac{\alpha}{2^{\alpha-1}\left(\ell_{2}-\ell_{1}\right)^{2}} \lambda\left(y_{1}+y_{2}-\frac{\ell_{1}+\ell_{2}}{2}\right) \\
& +\frac{\alpha(\alpha-1)}{\left(\ell_{2}-\ell_{1}\right)^{2}} \int_{0}^{1 / 2} \zeta^{\alpha-2} \lambda\left(y_{1}+y_{2}-\left((1-\zeta) \ell_{1}+\zeta \ell_{2}\right)\right) \mathrm{d} \zeta \\
& =-\frac{1}{2^{\alpha}\left(\ell_{2}-\ell_{1}\right)} \lambda^{\prime}\left(y_{1}+y_{2}-\frac{\ell_{1}+\ell_{2}}{2}\right)-\frac{\alpha}{2^{\alpha-1}\left(\ell_{2}-\ell_{1}\right)^{2}} \lambda\left(y_{1}+y_{2}-\frac{\ell_{1}+\ell_{2}}{2}\right) \\
& +\frac{\Gamma(\alpha+1)}{\left(\ell_{2}-\ell_{1}\right)^{\alpha+1}}\left(I_{\psi^{-1}\left(y_{1}+y_{2}-\left(\left(\ell_{1}+\ell_{2}\right) / 2\right)\right)^{+}}^{\alpha-1: \psi}\left[\lambda \circ \psi\left(\psi^{-1}\left(y_{1}+y_{2}-\ell_{1}\right)\right)\right]\right. \\
& I_{2}=\int_{1 / 2}^{1}(1-\zeta)^{\alpha} \lambda^{\prime \prime}\left(y_{1}+y_{2}-\left(\zeta \ell_{2}+(1-\zeta) \ell_{1}\right)\right) \mathrm{d} \zeta \\
& =\frac{1}{2^{\alpha}\left(\ell_{2}-\ell_{1}\right)} \lambda^{\prime}\left(y_{1}+y_{2}-\frac{\ell_{1}+\ell_{2}}{2}\right) \\
& -\frac{\alpha}{\ell_{2}-\ell_{1}} \int_{1 / 2}^{1}(1-\zeta)^{\alpha-1} \lambda^{\prime}\left(y_{1}+y_{2}-\left((1-\zeta) \ell_{1}+\zeta \ell_{2}\right)\right) \mathrm{d} \zeta \\
& =\frac{1}{2^{\alpha}\left(\ell_{2}-\ell_{1}\right)} \lambda^{\prime}\left(y_{1}+y_{2}-\frac{\ell_{1}+\ell_{2}}{2}\right)+\frac{\alpha}{2^{\alpha-1}\left(\ell_{2}-\ell_{1}\right)^{2}} \lambda\left(y_{1}+y_{2}-\frac{\ell_{1}+\ell_{2}}{2}\right) \\
& +\frac{\alpha(\alpha-1)}{\left(\ell_{2}-\ell_{1}\right)^{2}} \int_{1 / 2}^{1}(1-\zeta)^{\alpha-2} \lambda\left(y_{1}+y_{2}-\left((1-\zeta) \ell_{1}+\zeta \ell_{2}\right)\right) \mathrm{d} \zeta \\
& =\frac{1}{2^{\alpha}\left(\ell_{2}-\ell_{1}\right)} \lambda^{\prime}\left(y_{1}+y_{2}-\frac{\ell_{1}+\ell_{2}}{2}\right)-\frac{\alpha}{2^{\alpha-1}\left(\ell_{2}-\ell_{1}\right)^{2}} \lambda\left(y_{1}+y_{2}-\frac{\ell_{1}+\ell_{2}}{2}\right) \\
& +\frac{\Gamma(\alpha+1)}{\left(\ell_{2}-\ell_{1}\right)^{\alpha+1}}\left(I_{\psi^{-1}\left(y_{1}+y_{2}-\left(\left(\ell_{1}+\ell_{2}\right) / 2\right)\right)^{-}}^{\alpha-\psi}\left[\lambda \circ \psi\left(\psi^{-1}\left(y_{1}+y_{2}-\ell_{2}\right)\right)\right]\right. \text {. }
\end{aligned}
$$

Substituting (91) and (92) in (90), we get (89).

Corollary 6. If we set $\ell_{1}=y_{1}$ and $\ell_{2}=y_{2}$ in Lemma 6, we get 


$$
\begin{aligned}
& \frac{2^{\alpha-2} \Gamma(\alpha)}{\left(y_{2}-y_{1}\right)^{\alpha-1}}\left\{\left(I_{\psi^{-1}}^{\alpha: \psi}\left(\left(y_{1}+y_{2}\right) / 2\right)^{+}\right)\left(\lambda \circ \psi\left(\psi^{-1}\left(y_{2}\right)\right)\right)\right. \\
& \left.\quad+\left(I_{\psi^{-1}}^{\alpha: \psi}\left(\left(y_{1}+y_{2}\right) / 2\right)^{-}\right)\left(\lambda \circ \psi\left(\psi^{-1}\left(y_{1}\right)\right)\right)\right\}-\lambda\left(\frac{y_{1}+y_{2}}{2}\right) \\
& =\frac{\left(y_{2}-y_{1}\right)^{2}}{\alpha \cdot 2^{2-\alpha}}\left[\int_{0}^{1 / 2} \zeta^{\alpha} \lambda^{\prime \prime}\left(\zeta y_{1}+(1-\zeta) y_{2}\right) \mathrm{d} \zeta+\int_{1 / 2}^{1}(1-\zeta)^{\alpha} \lambda^{\prime \prime}\left(\zeta y_{1}+(1-\zeta) y_{2}\right) \mathrm{d} \zeta\right]
\end{aligned}
$$

Corollary 7. If we set $\psi(\gamma)=\gamma$ in Lemma 6, we get

$$
\begin{aligned}
& \frac{2^{\alpha-2} \Gamma(\alpha)}{\left(\ell_{2}-\ell_{1}\right)^{\alpha-1}}\left\{\left(J_{\left.\left(y_{1}+y_{2}-\left(\left(\ell_{1}+\ell_{2}\right) / 2\right)\right)^{+}\right)\left(\lambda\left(y_{1}+y_{2}-\ell_{1}\right)\right)}^{\alpha}\right)\left(\lambda\left(y_{1}+y_{2}-\ell_{2}\right)\right)\right\}-\lambda\left(y_{1}+y_{2}-\frac{\ell_{1}+\ell_{2}}{2}\right) \\
& \quad+\left(J_{\left(y_{1}+y_{2}-\left(\left(\ell_{1}+\ell_{2}\right) / 2\right)\right)^{-}}^{\alpha}\right) \\
& =\frac{\left(\ell_{2}-\ell_{1}\right)^{2}}{\alpha \cdot 2^{2-\alpha}}\left[\int_{0}^{1 / 2} \zeta^{\alpha} \lambda^{\prime \prime}\left(y_{1}+y_{2}-\left(\zeta \ell_{2}+(1-\zeta) \ell_{1}\right)\right) \mathrm{d} \zeta\right. \\
& \left.\quad+\int_{1 / 2}^{1}(1-\zeta)^{\alpha} \lambda^{\prime \prime}\left(y_{1}+y_{2}-\left(\zeta \ell_{2}+(1-\zeta) \ell_{1}\right)\right) \mathrm{d} \zeta\right]
\end{aligned}
$$

Moreover, if we set $\ell_{1}=y_{1}$ and $\ell_{2}=y_{2}$, we obtain Lemma 2.1 of [34] for $m=1$.
Theorem 12. If $\left(A_{1}\right)$ is satisfied and $\left|\lambda^{\prime \prime}\right|$ is a convex function on $\left[y_{1}, y_{2}\right]$, then

$$
\begin{aligned}
& \mid \frac{2^{\alpha-2} \Gamma(\alpha)}{\left(\ell_{2}-\ell_{1}\right)^{\alpha-1}}\left\{\left(I_{\psi^{-1}\left(y_{1}+y_{2}-\left(\left(\ell_{1}+\ell_{2}\right) / 2\right)\right)^{+}}^{\alpha-1: \psi}\left(\lambda \circ \psi\left(\psi^{-1}\left(y_{1}+y_{2}-\ell_{1}\right)\right)\right)\right.\right. \\
& \quad+\left(I_{\psi^{-1}\left(y_{1}+y_{2}-\left(\left(\ell_{1}+\ell_{2}\right) / 2\right)\right)^{-}}^{\alpha-1: \psi}\left(\lambda \circ \psi\left(\psi^{-1}\left(y_{1}+y_{2}-\ell_{2}\right)\right)\right)\right\}-\lambda\left(y_{1}+y_{2}-\frac{\ell_{1}+\ell_{2}}{2}\right) \mid \\
& \leq \frac{\left(\ell_{2}-\ell_{1}\right)^{2}}{4 \alpha(\alpha+1)}\left\{\left|\lambda^{\prime \prime}\left(y_{1}\right)\right|+\left|\lambda^{\prime \prime}\left(y_{2}\right)\right|-\frac{\left|\lambda^{\prime \prime}\left(\ell_{1}\right)\right|+\left|\lambda^{\prime \prime}\left(\ell_{2}\right)\right|}{2}\right\} .
\end{aligned}
$$

Proof. By using Lemma 6, properties of modulus, and Jensen-Mercer inequality, we have 


$$
\begin{aligned}
& \mid \frac{2^{\alpha-2} \Gamma(\alpha)}{\left(\ell_{2}-\ell_{1}\right)^{\alpha-1}}\left\{\left(I_{\psi^{-1}\left(y_{1}+y_{2}-\left(\left(\ell_{1}+\ell_{2}\right) / 2\right)\right)^{+}}^{\alpha-1}\right)\left(\lambda \circ \psi\left(\psi^{-1}\left(y_{1}+y_{2}-\ell_{1}\right)\right)\right)\right. \\
& \left.\quad+\left(I_{\psi^{-1}\left(y_{1}+y_{2}-\left(\left(\ell_{1}+\ell_{2}\right) / 2\right)\right)^{-}}^{\alpha-1: \psi}\right)\left(\lambda \circ \psi\left(\psi^{-1}\left(y_{1}+y_{2}-\ell_{2}\right)\right)\right)\right\}-\lambda\left(y_{1}+y_{2}-\frac{\ell_{1}+\ell_{2}}{2}\right) \mid \\
& \leq \frac{\left(\ell_{2}-\ell_{1}\right)^{2}}{\alpha \cdot 2^{2-\alpha}}\left[\int_{0}^{1 / 2} \zeta^{\alpha}\left|\lambda^{\prime \prime}\left(y_{1}+y_{2}-\left((1-\zeta) \ell_{1}+\zeta \ell_{2}\right)\right)\right| \mathrm{d} \zeta\right. \\
& \left.\quad+\int_{1 / 2}^{1}(1-\zeta)^{\alpha}\left|\lambda^{\prime \prime}\left(y_{1}+y_{2}-\left((1-\zeta) \ell_{1}+\zeta \ell_{2}\right)\right)\right| \mathrm{d} \zeta\right] \\
& \leq \frac{\left(\ell_{2}-\ell_{1}\right)^{2}}{\alpha \cdot 2^{2-\alpha}}\left[\int_{0}^{1 / 2} \zeta^{\alpha}\left\{\left|\lambda^{\prime \prime}\left(y_{1}\right)\right|+\left|\lambda^{\prime \prime}\left(y_{2}\right)\right|-\left((1-\zeta)\left|\lambda^{\prime \prime}\left(\ell_{1}\right)\right|+\zeta\left|\lambda^{\prime \prime}\left(\ell_{2}\right)\right|\right)\right\} \mathrm{d} \zeta\right. \\
& \left.\quad+\int_{1 / 2}^{1}(1-\zeta)^{\alpha}\left\{\left|\lambda^{\prime \prime}\left(y_{1}\right)\right|+\left|\lambda^{\prime \prime}\left(y_{2}\right)\right|-\left((1-\zeta)\left|\lambda^{\prime \prime}\left(\ell_{1}\right)\right|+\zeta\left|\lambda^{\prime \prime}\left(\ell_{2}\right)\right|\right)\right\} \mathrm{d} \zeta\right]
\end{aligned}
$$

and after integration, we get required result.

Corollary 8. If we set $\ell_{1}=y_{1}$ and $\ell_{2}=y_{2}$ in Theorem 12, we get

$$
\begin{aligned}
& \mid \frac{2^{\alpha-2} \Gamma(\alpha)}{\left(y_{2}-y_{1}\right)^{\alpha-1}}\left\{\left(I_{\psi^{-1}\left(\left(y_{1}+y_{2}\right) / 2\right)^{+}}^{\alpha-1:}\right)\left(\lambda \circ \psi\left(\psi^{-1}\left(y_{2}\right)\right)\right)\right. \\
& +\left(I_{\psi^{-1}\left(\left(y_{1}+y_{2}\right) / 2\right)^{-}}^{\alpha-1:}\right)\left(\lambda \circ \psi\left(\psi^{-1}\left(y_{1}\right)\right)\right)-\lambda\left(\frac{y_{1}+y_{2}}{2}\right) \mid \\
& \leq \frac{\left(y_{2}-y_{1}\right)^{2}}{8 \alpha(\alpha+1)}\left(\left|\lambda^{\prime \prime}\left(y_{1}\right)\right|+\left|\lambda^{\prime \prime}\left(y_{2}\right)\right|\right) .
\end{aligned}
$$

Corollary 9. If we set $\psi(\gamma)=\gamma$ in Theorem 12, we get

$$
\begin{aligned}
& \mid \frac{2^{\alpha-2} \Gamma(\alpha)}{\left(\ell_{2}-\ell_{1}\right)^{\alpha-1}}\left\{\left(J_{\left(y_{1}+y_{2}-\left(\left(\ell_{1}+\ell_{2}\right) / 2\right)\right)^{+}}^{\alpha-1}\right)\left(\lambda\left(y_{1}+y_{2}-\ell_{1}\right)\right)\right. \\
& \quad+\left(J_{\left(y_{1}+y_{2}-\left(\left(\ell_{1}+\ell_{2}\right) / 2\right)\right)^{-}}^{\alpha-1}\right)\left(\lambda\left(y_{1}+y_{2}-\ell_{2}\right)\right)-\lambda\left(y_{1}+y_{2}-\frac{\ell_{1}+\ell_{2}}{2}\right) \mid \\
& \leq \frac{\left(\ell_{2}-\ell_{1}\right)^{2}}{4 \alpha(\alpha+1)}\left\{\left|\lambda^{\prime \prime}\left(y_{1}\right)\right|+\left|\lambda^{\prime \prime}\left(y_{2}\right)\right|-\left(\frac{\left|\lambda^{\prime \prime}\left(\ell_{1}\right)\right|+\left|\lambda^{\prime \prime}\left(\ell_{2}\right)\right|}{2}\right)\right\} .
\end{aligned}
$$

Remark 22. If we set $\psi(\gamma)=\gamma, \ell_{1}=y_{1}$, and $\ell_{2}=y_{2}$ in Theorem 12, we get Theorem 2.1 of [34].
Moreover, if we set $\alpha=2$, we obtain Proposition 1 of [33]. 
Theorem 13. If $\left(A_{1}\right)$ is satisfied and $\left|\lambda^{\prime \prime}\right|^{q}$ is convex function,

then

$$
\begin{aligned}
& \mid \frac{2^{\alpha-2} \Gamma(\alpha)}{\left(\ell_{2}-\ell_{1}\right)^{\alpha-1}}\left(\left(I_{\psi^{-1}\left(y_{1}+y_{2}-\left(\left(\ell_{1}+\ell_{2}\right) / 2\right)\right)^{+}}^{\alpha-1: \psi}\right)\left(\lambda \circ \psi\left(\psi^{-1}\left(y_{1}+y_{2}-\ell_{1}\right)\right)\right)\right) \\
& \quad+\left(I_{\psi^{-1}\left(y_{1}+y_{2}-\left(\left(\ell_{1}+\ell_{2}\right) / 2\right)\right)^{-}}^{\alpha-1: \psi}\right)\left(\lambda \circ \psi\left(\psi^{-1}\left(y_{1}+y_{2}-\ell_{2}\right)\right)\right)-\lambda\left(y_{1}+y_{2}-\frac{\ell_{1}+\ell_{2}}{2}\right) \mid \\
& \leq \frac{\left(\ell_{2}-\ell_{1}\right)^{2}}{\alpha \cdot 2^{2-\alpha}}\left(\frac{1}{2^{p \alpha+1}(p \alpha+1)}\right)^{1 / p}\left[\left(\frac{\left|\lambda^{\prime \prime}\left(y_{1}\right)\right|^{q}+\left|\lambda^{\prime \prime}\left(y_{2}\right)\right|^{q}}{2}-\frac{3\left|\lambda^{\prime \prime}\left(\ell_{1}\right)\right|^{q}+\left|\lambda^{\prime \prime}\left(\ell_{2}\right)\right|^{q}}{8}\right)^{1 / q}\right. \\
& \quad+\left(\frac{\left|\lambda^{\prime \prime}\left(y_{1}\right)\right|^{q}+\left|\lambda^{\prime \prime}\left(y_{2}\right)\right|^{q}}{2}-\frac{\left|\lambda^{\prime \prime}\left(\ell_{1}\right)\right|^{q}+3\left|\lambda^{\prime \prime}\left(\ell_{2}\right)\right|^{q}}{8}\right)^{1 / q},
\end{aligned}
$$

where $q>1$ and $(1 / p)+(1 / q)=1$ for all $\ell_{1}, \ell_{2} \in\left[y_{1}, y_{2}\right]$.

Proof. Applying Lemma 6, Hölder and Jensen-Mercer inequalities, the fact that $\left|\lambda^{\prime \prime}\right|^{q}$ is convex function, and properties of modulus, we have

$$
\begin{aligned}
& \mid \frac{2^{\alpha-2} \Gamma(\alpha)}{\left(\ell_{2}-\ell_{1}\right)^{\alpha-1}}\left(\left(I_{\psi^{-1}\left(y_{1}+y_{2}-\left(\left(\ell_{1}+\ell_{2}\right) / 2\right)\right)^{+}}^{\alpha-1: \psi}\right)\left(\lambda \circ \psi\left(\psi^{-1}\left(y_{1}+y_{2}-\ell_{1}\right)\right)\right)\right) \\
& +\left(I_{\psi^{-1}\left(y_{1}+y_{2}-\left(\left(\ell_{1}+\ell_{2}\right) / 2\right)\right)^{-}}^{\alpha-1:}\right)\left(\lambda \circ \psi\left(\psi^{-1}\left(y_{1}+y_{2}-\ell_{2}\right)\right)\right)-\lambda\left(y_{1}+y_{2}-\frac{\ell_{1}+\ell_{2}}{2}\right) \mid \\
& \leq \frac{\left(\ell_{2}-\ell_{1}\right)^{2}}{\alpha \cdot 2^{2-\alpha}}\left[\int_{0}^{1 / 2} \zeta^{\alpha}\left|\lambda^{\prime \prime}\left(y_{1}+y_{2}-\left((1-\zeta) \ell_{1}+\zeta \ell_{2}\right)\right)\right| \mathrm{d} \zeta\right. \\
& +\int_{1 / 2}^{1}(1-\zeta)^{\alpha}\left|\lambda^{\prime \prime}\left(y_{1}+y_{2}-\left((1-\zeta) \ell_{1}+\zeta \ell_{2}\right)\right)\right| \mathrm{d} \zeta \\
& \leq \frac{\left(\ell_{2}-\ell_{1}\right)^{2}}{\alpha \cdot 2^{2-\alpha}}\left[\left(\int_{0}^{1 / 2} \zeta^{p \alpha} \mathrm{d} \zeta\right)^{1 / p}\left(\int_{0}^{1 / 2}\left|\lambda^{\prime \prime}\left(y_{1}+y_{2}-\left((1-\zeta) \ell_{1}+\zeta \ell_{2}\right)\right)\right|^{q} \mathrm{~d} \zeta\right)^{1 / q}\right. \\
& +\left(\int_{1 / 2}^{1}(1-\zeta)^{p \alpha} \mathrm{d} \zeta\right)^{1 / p}\left(\int_{1 / 2}^{1}\left|\lambda^{\prime \prime}\left(y_{1}+y_{2}-\left((1-\zeta) \ell_{1}+\zeta \ell_{2}\right)\right)\right|^{q} \mathrm{~d} \zeta\right)^{1 / q} \\
& \leq \frac{\left(\ell_{2}-\ell_{1}\right)^{2}}{\alpha \cdot 2^{2-\alpha}}\left(\frac{1}{2^{p \alpha+1}(p \alpha+1)}\right)^{1 / p} \\
& \times\left[\left(\int_{0}^{1 / 2}\left(\left|\lambda^{\prime \prime}\left(y_{1}\right)\right|^{q}+\left|\lambda^{\prime \prime}\left(y_{2}\right)\right|^{q}-(1-\zeta)\left|\lambda^{\prime \prime}\left(\ell_{1}\right)\right|^{q}-\zeta\left|\lambda^{\prime \prime}\left(\ell_{2}\right)\right|^{q}\right) \mathrm{d} \zeta\right)^{1 / q}\right. \\
& +\left(\int_{1 / 2}^{1}\left(\left|\lambda^{\prime \prime}\left(y_{1}\right)\right|^{q}+\left|\lambda^{\prime \prime}\left(y_{2}\right)\right|^{q}-(1-\zeta)\left|\lambda^{\prime \prime}\left(\ell_{1}\right)\right|^{q}-\zeta\left|\lambda^{\prime \prime}\left(\ell_{2}\right)\right|^{q}\right) \mathrm{d} \zeta\right)^{1 / q} \\
& =\frac{\left(\ell_{2}-\ell_{1}\right)^{2}}{\alpha \cdot 2^{2-\alpha}}\left(\frac{1}{2^{p \alpha+1}(p \alpha+1)}\right)^{1 / p}\left[\left(\frac{\left|\lambda^{\prime \prime}\left(y_{1}\right)\right|^{q}+\left|\lambda^{\prime \prime}\left(y_{2}\right)\right|^{q}}{2}-\frac{3\left|\lambda^{\prime \prime}\left(\ell_{1}\right)\right|^{q}+\left|\lambda^{\prime \prime}\left(\ell_{2}\right)\right|^{q}}{8}\right)^{1 / q}\right. \\
& +\left(\frac{\left|\lambda^{\prime \prime}\left(y_{1}\right)\right|^{q}+\left|\lambda^{\prime \prime}\left(y_{2}\right)\right|^{q}}{2}-\frac{\left|\lambda^{\prime \prime}\left(\ell_{1}\right)\right|^{q}+3\left|\lambda^{\prime \prime}\left(\ell_{2}\right)\right|^{q}}{8}\right)^{1 / q} \text {. }
\end{aligned}
$$


Corollary 10. If we set $\ell_{1}=y_{1}$ and $\ell_{2}=y_{2}$ in Theorem 12, we get

$$
\begin{aligned}
& \left.\mid \frac{2^{\alpha-2} \Gamma(\alpha)}{\left(y_{2}-y_{1}\right)^{\alpha-1}}\left(\left(I_{\psi^{-1}}^{\alpha-1: \psi}\left(y_{1}+y_{2}\right) / 2\right)^{+}\right)\left(\lambda \circ \psi\left(\psi^{-1}\left(y_{2}\right)\right)\right)\right) \\
& \quad+\left(I_{\psi^{-1}\left(\left(y_{1}+y_{2}\right) / 2\right)^{-}}^{\alpha-1: \psi}\right)\left(\lambda \circ \psi\left(\psi^{-1}\left(y_{1}\right)\right)\right)-\lambda\left(\frac{y_{1}+y_{2}}{2}\right) \mid \\
& \leq \frac{\left(\ell_{2}-\ell_{1}\right)^{2}}{\alpha \cdot 2^{2-\alpha}}\left(\frac{1}{2^{p \alpha+1}(p \alpha+1)}\right)^{1 / p}\left[\left(\frac{\left|\lambda^{\prime \prime}\left(y_{1}\right)\right|^{q}+3\left|\lambda^{\prime \prime}\left(y_{2}\right)\right|^{q}}{8}\right)^{1 / q}\right. \\
& \quad+\left(\frac{3\left|\lambda^{\prime \prime}\left(y_{1}\right)\right|^{q}+\left|\lambda^{\prime \prime}\left(y_{2}\right)\right|^{q}}{8}\right)^{1 / q} .
\end{aligned}
$$

Corollary 11. If we set $\psi(\gamma)=\gamma$ in Theorem 12, we get

$$
\begin{aligned}
& \mid \frac{2^{\alpha-2} \Gamma(\alpha)}{\left(\ell_{2}-\ell_{1}\right)^{\alpha-1}}\left(\left(J_{\left.\left.\left(y_{1}+y_{2}-\left(\left(\ell_{1}+\ell_{2}\right) / 2\right)\right)^{+}\right)\left(\lambda\left(y_{1}+y_{2}-\ell_{1}\right)\right)\right)}\right.\right. \\
& \quad+\left(J_{\left.\left(y_{1}+y_{2}-\left(\left(\ell_{1}+\ell_{2}\right) / 2\right)\right)^{-}\right)\left(\lambda\left(y_{1}+y_{2}-\ell_{2}\right)\right)}^{\alpha-1}\right) \\
& \quad-\lambda\left(y_{1}+y_{2}-\frac{\ell_{1}+\ell_{2}}{2}\right) \mid \\
& \leq \frac{\left(\ell_{2}-\ell_{1}\right)^{2}}{\alpha \cdot 2^{2-\alpha}}\left(\frac{1}{2^{p \alpha+1}(p \alpha+1)}\right)^{1 / p}\left[\left(\frac{\left|\lambda^{\prime \prime}\left(y_{1}\right)\right|^{q}+\left|\lambda^{\prime \prime}\left(y_{2}\right)\right|^{q}}{2}-\frac{3\left|\lambda^{\prime \prime}\left(\ell_{1}\right)\right|^{q}+\left|\lambda^{\prime \prime}\left(\ell_{2}\right)\right|^{q}}{8}\right)^{1 / q}\right. \\
& \quad+\left(\frac{\left|\lambda^{\prime \prime}\left(y_{1}\right)\right|^{q}+\left|\lambda^{\prime \prime}\left(y_{2}\right)\right|^{q}}{2}-\frac{\left|\lambda^{\prime \prime}\left(\ell_{1}\right)\right|^{q}+3\left|\lambda^{\prime \prime}\left(\ell_{2}\right)\right|^{q}}{8}\right)^{1 / q} \cdot
\end{aligned}
$$

Theorem 14. If $\left(A_{1}\right)$ is satisfied and $\left|\lambda^{\prime \prime}\right|^{q}$ is convex function, then

$$
\begin{aligned}
& \mid \frac{2^{\alpha-2} \Gamma(\alpha)}{\left(\ell_{2}-\ell_{1}\right)^{\alpha-1}}\left(\left(I_{\psi^{-1}\left(y_{1}+y_{2}-\left(\left(\ell_{1}+\ell_{2}\right) / 2\right)\right)^{+}}^{\alpha-1: \psi}\right)\left(\lambda \circ \psi\left(\psi^{-1}\left(y_{1}+y_{2}-\ell_{1}\right)\right)\right)\right) \\
& \quad+\left(I_{\psi^{-1}\left(y_{1}+y_{2}-\left(\left(\ell_{1}+\ell_{2}\right) / 2\right)\right)^{-}}^{\alpha-1: \psi}\right)\left(\lambda \circ \psi\left(\psi^{-1}\left(y_{1}+y_{2}-\ell_{2}\right)\right)\right)-\lambda\left(y_{1}+y_{2}-\frac{\ell_{1}+\ell_{2}}{2}\right) \mid \\
& \leq \frac{\left(\ell_{2}-\ell_{1}\right)^{2}}{\alpha \cdot 2^{2-\alpha}}\left(\frac{1}{2^{\alpha+1}(\alpha+1)}\right)^{1-(1 / q)} \\
& \quad \times\left[\left(\frac{\left|\lambda^{\prime \prime}\left(y_{1}\right)\right|^{q}+\left|\lambda^{\prime \prime}\left(y_{2}\right)\right|^{q}}{2^{\alpha+1}(\alpha+1)}-\frac{(\alpha+3)\left|\lambda^{\prime \prime}\left(\ell_{1}\right)\right|^{q}}{2^{\alpha+2}(\alpha+1)(\alpha+2)}-\frac{\left|\lambda^{\prime \prime}\left(\ell_{2}\right)\right|^{q}}{2^{\alpha+2}(\alpha+2)}\right)^{1 / q}\right. \\
& \quad+\left(\frac{\left|\lambda^{\prime \prime}\left(y_{1}\right)\right|^{q}+\left|\lambda^{\prime \prime}\left(y_{2}\right)\right|^{q}}{2^{\alpha+1}(\alpha+1)}-\frac{\left|\lambda^{\prime \prime}\left(\ell_{1}\right)\right|^{q}}{2^{\alpha+2}(\alpha+2)}-\frac{(\alpha+3)\left|\lambda^{\prime \prime}\left(\ell_{2}\right)\right|^{q}}{2^{\alpha+2}(\alpha+1)(\alpha+2)}\right)^{1 / q},
\end{aligned}
$$


where $q \geq 1$ for all $\ell_{1}, \ell_{2} \in\left[y_{1}, y_{2}\right]$.

Proof. From Lemma 2, power-mean and Jensen-Mercer inequalities, the fact that $\left|\lambda^{\prime \prime}\right|^{q}$ is convex function, and properties of modulus, we have

$$
\begin{aligned}
& \mid \frac{2^{\alpha-2} \Gamma(\alpha)}{\left(\ell_{2}-\ell_{1}\right)^{\alpha-1}}\left(\left(I_{\psi^{-1}\left(y_{1}+y_{2}-\left(\left(\ell_{1}+\ell_{2}\right) / 2\right)\right)^{+}}^{\alpha-1:{ }^{+}}\right)\left(\lambda \circ \psi\left(\psi^{-1}\left(y_{1}+y_{2}-\ell_{1}\right)\right)\right)\right) \\
& +\left(I_{\psi^{-1}\left(y_{1}+y_{2}-\left(\left(\ell_{1}+\ell_{2}\right) / 2\right)\right)^{-}}^{\alpha-1}\right)\left(\lambda \circ \psi\left(\psi^{-1}\left(y_{1}+y_{2}-\ell_{2}\right)\right)\right)-\lambda\left(y_{1}+y_{2}-\frac{\ell_{1}+\ell_{2}}{2}\right) \mid \\
& \leq \frac{\left(\ell_{2}-\ell_{1}\right)^{2}}{\alpha \cdot 2^{2-\alpha}}\left[\int_{0}^{1 / 2} \zeta^{\alpha}\left|\lambda^{\prime \prime}\left(y_{1}+y_{2}-\left((1-\zeta) \ell_{1}+\zeta \ell_{2}\right)\right)\right| \mathrm{d} \zeta\right. \\
& +\int_{1 / 2}^{1}(1-\zeta)^{\alpha}\left|\lambda^{\prime \prime}\left(y_{1}+y_{2}-\left((1-\zeta) \ell_{1}+\zeta \ell_{2}\right)\right)\right| \mathrm{d} \zeta \\
& \leq \frac{\left(\ell_{2}-\ell_{1}\right)^{2}}{\alpha \cdot 2^{2-\alpha}}\left[\left(\int_{0}^{1 / 2} \zeta^{\alpha} \mathrm{d} \zeta\right)^{1-(1 / q)}\right. \\
& \times\left(\left(\left|\lambda^{\prime \prime}\left(y_{1}\right)\right|^{q}+\left|\lambda^{\prime \prime}\left(y_{2}\right)\right|^{q}\right) \int_{0}^{1 / 2} \zeta^{\alpha} \mathrm{d} \zeta-\left|\lambda^{\prime \prime}\left(\ell_{1}\right)\right|^{q} \int_{0}^{1 / 2} \zeta^{\alpha}(1-\zeta) \mathrm{d} \zeta-\left|\lambda^{\prime \prime}\left(\ell_{2}\right)\right|^{q} \int_{0}^{1 / 2} \zeta^{\alpha+1} \mathrm{~d} \zeta\right)^{1 / q} \\
& +\left(\int_{1 / 2}^{1}(1-\zeta)^{\alpha} \mathrm{d} \zeta\right)^{1-(1 / q)} \\
& \times\left(\left(\left|\lambda^{\prime \prime}\left(y_{1}\right)\right|^{q}+\left|\lambda^{\prime \prime}\left(y_{2}\right)\right|^{q}\right) \int_{1 / 2}^{1}(1-\zeta)^{\alpha} \mathrm{d} \zeta-\left|\lambda^{\prime \prime}\left(\ell_{1}\right)\right|^{q} \int_{1 / 2}^{1} \zeta(1-\zeta)^{\alpha} \mathrm{d} \zeta-\left|\lambda^{\prime \prime}\left(\ell_{2}\right)\right|^{q} \int_{1 / 2}^{1}(1-\zeta)^{\alpha+1} \mathrm{~d} \zeta\right)^{1 / q} \\
& =\frac{\left(\ell_{2}-\ell_{1}\right)^{2}}{\alpha \cdot 2^{2-\alpha}}\left(\frac{1}{2^{\alpha+1}(\alpha+1)}\right)^{1-(1 / q)} \\
& \times\left[\left(\frac{\left|\lambda^{\prime \prime}\left(y_{1}\right)\right|^{q}+\left|\lambda^{\prime \prime}\left(y_{2}\right)\right|^{q}}{2^{\alpha+1}(\alpha+1)}-\frac{(\alpha+3)\left|\lambda^{\prime \prime}\left(\ell_{1}\right)\right|^{q}}{2^{\alpha+2}(\alpha+1)(\alpha+2)}-\frac{\left|\lambda^{\prime \prime}\left(\ell_{2}\right)\right|^{q}}{2^{\alpha+2}(\alpha+2)}\right)^{1 / q}\right. \\
& +\left(\frac{\left|\lambda^{\prime \prime}\left(y_{1}\right)\right|^{q}+\left|\lambda^{\prime \prime}\left(y_{2}\right)\right|^{q}}{2^{\alpha+1}(\alpha+1)}-\frac{\left|\lambda^{\prime \prime}\left(\ell_{1}\right)\right|^{q}}{2^{\alpha+2}(\alpha+2)}-\frac{(\alpha+3)\left|\lambda^{\prime \prime}\left(\ell_{2}\right)\right|^{q}}{2^{\alpha+2}(\alpha+1)(\alpha+2)}\right)^{1 / q} \text {. }
\end{aligned}
$$

Remark 23. If we set $\psi(\gamma)=\gamma, \ell_{1}=y_{1}$, and $\ell_{2}=y_{2}$ in Theorem 14, we get Theorem 2.2 of [34] for $m=s=1$.

Moreover, if we set $\alpha=2$, we obtain Proposition 5 of [33].

\section{Application}

In this last section, we will give an application of our results using modified Bessel function of the first kind.

Let the function $\mathfrak{\Im}_{p}: \mathfrak{R} \longrightarrow[1,+\infty)$ be defined by

$$
\mathfrak{\Im}_{p}\left(\ell_{1}\right)=2^{p} \Gamma(p+1) \ell_{1}^{-v} I_{p}\left(\ell_{1}\right), \quad p>1, \ell_{1} \in \mathfrak{R} .
$$

For this, we recall the modified Bessel function of the first kind $\mathfrak{\Im}_{p}$ which is defined as follows [35]:

$$
\mathfrak{\Im}_{p}\left(\ell_{1}\right)=\sum_{n \geq 0} \frac{\left(\ell_{1} / 2\right)^{p+2 n}}{n ! \Gamma(p+n+1)} .
$$

The first and the $n$th order derivative formula of $\mathfrak{\Im}_{p}$ is, respectively, given by the following [36]: 


$$
\begin{aligned}
\mathfrak{\Im}_{p}^{\prime}\left(\ell_{1}\right)= & \frac{\ell_{1}}{2(p+1)} \Im_{p+1}\left(\ell_{1}\right), \\
\frac{\mathrm{d}^{n} \Im_{p}\left(\ell_{1}\right)}{\mathrm{d} \ell_{1}^{n}}= & 2^{n-2 p} \sqrt{\pi} \ell_{1}^{p-n} \Gamma(p+1) \\
& \times{ }_{2} F_{3}\left(\frac{p+1}{2}, \frac{p+2}{2} ; \frac{p+1-n}{2}, \frac{p+2-n}{2}, p+1 ; \frac{\ell_{1}^{2}}{4}\right),
\end{aligned}
$$

where ${ }_{2} F_{3}(\cdot, \cdot ; \cdot, \cdot, \cdot ; \cdot)$ is the hypergeometric function defined by the following [36]:

$$
\begin{aligned}
& { }_{2} F_{3}\left(\frac{p+1}{2}, \frac{p+2}{2} ; \frac{p+1-n}{2}, \frac{p+2-n}{2}, p+1 ; \frac{\ell_{1}^{2}}{4}\right) \\
& =\sum_{k=0}^{\infty} \frac{((p+1) / 2)_{k}((p+2) / 2)_{k}}{((p-1) / 2)_{k}((p-2) / 2)_{k}(p+1)_{k}} \cdot \frac{\ell_{1}^{2 k}}{4^{k} \cdot k !},
\end{aligned}
$$

and for some parameter $v$, the Pochhammer symbol $(v)_{k}$ is defined as

$$
\begin{aligned}
& (v)_{0}=1, \\
& (v)_{k}=v(v+1) \cdots(v+k+1), \quad k=1,2,3, \ldots
\end{aligned}
$$

Proposition 1. Let $0<y_{1}<y_{2}$ be real numbers and $p>-1$, then

$$
\begin{aligned}
& \left|\frac{\mathfrak{J}_{p}\left(y_{2}\right)-\mathfrak{J}_{p}\left(y_{1}\right)}{y_{2}-y_{1}}-\frac{y_{1}+y_{2}}{4(p+1)} \mathfrak{J}_{p+1}\left(\frac{y_{1}+y_{2}}{2}\right)\right| \\
& \leq \frac{\left(y_{2}-y_{1}\right)^{2}}{48} 2^{3-2 p} \sqrt{\pi} \Gamma(p+1) \\
& \times\left\{y_{1}^{p-3} \cdot{ }_{2} F_{3}\left(\frac{p+1}{2}, \frac{p+2}{2} ; \frac{p-2}{2}, \frac{p-1}{2}, p+1 ; \frac{y_{1}^{2}}{4}\right)\right. \\
& +y_{2}^{p-3} \cdot{ }_{2} F_{3}\left(\frac{p+1}{2}, \frac{p+2}{2} ; \frac{p-2}{2}, \frac{p-1}{2}, p+1 ; \frac{y_{2}^{2}}{4}\right) \text {. } \\
& \leq \frac{\left(y_{2}-y_{1}\right)^{2}}{48} 2^{3-2 p} \sqrt{\pi} \Gamma(p+1) \\
& \times\left\{y_{1}^{p-3} \cdot{ }_{2} F_{3}\left(\frac{p+1}{2}, \frac{p+2}{2} ; \frac{p-2}{2}, \frac{p-1}{2}, p+1 ; \frac{y_{1}^{2}}{4}\right)\right. \\
& +y_{2}^{p-3} \cdot{ }_{2} F_{3}\left(\frac{p+1}{2}, \frac{p+2}{2} ; \frac{p-2}{2}, \frac{p-1}{2}, p+1 ; \frac{y_{2}^{2}}{4}\right) .
\end{aligned}
$$

Proof. Let $\lambda\left(\ell_{1}\right)=\mathfrak{\Im}_{p}^{\prime}\left(\ell_{1}\right)$. Note that the function $\ell_{1} \longrightarrow \mathfrak{I}^{\prime \prime \prime}\left(\ell_{1}\right)$ is convex on the interval $[0,+\infty)$ for each $p>-1$. Using Corollary 3 and relations (107) and (108), we obtain the desired inequality (111).
Remark 24. Using the same technique like Proposition 1, we can obtain some new interesting inequalities pertaining modified Bessel function of the first kind or the well-known $q$-digamma function for $q \in(0,1)$ from our generic results. We omit here their proofs, and the details are left to the interested reader.

\section{Conclusion}

In this article, some new Hermite-Jensen-Mercer type inequalities involving $\psi$-Riemann-Liouville fractional integrals are found. Several $\psi$-Riemann-Liouville fractional integral inequalities using identities as auxiliary results are provided, and the known results are recaptured as special cases as well. Finally, the efficiency of our results is showed with an application via modified Bessel function of the first kind. We hope that current work using our idea and technique will attract the attention of researchers working in mathematical analysis and other related fields in pure and applied sciences.

\section{Data Availability}

No data were used to support this study.

\section{Conflicts of Interest}

The authors declare that they have no conflicts of interest.

\section{References}

[1] R. Hilfer, Applications of Fractional Calculus in Physics, World Scientific, vol. 35, pp. 87-130, Singapore, 2000.

[2] C. Cesarano, N. Pierpaolo, and E. R. Paolo, "Pseudo-Lucas functions of fractional degree and applications," Axioms, vol. 10, p. 51, 2021.

[3] J. Sabatier, O. P. Agrawal, and J. A. T. Machado, Advances in Fractional Calculus, Springer, Dordrecht, The Netherlands, 2007.

[4] M. S. Al-Luhaibi, "An analytical treatment to fractional Fornberg-Whitham equation," Mathematical Sciences, vol. 11, no. 1, pp. 1-6, 2017.

[5] S. Rashid, M. A. Latif, Z. Hammouch, and Y.-M. Chu, "Fractional integral inequalities for strongly $h$-preinvex functions for a kth order differentiable functions," Symmetry, vol. 11, no. 12, p. 1448, 2019.

[6] T. Abdeljawad, S. Rashid, Z. Hammouch, İ. İşcan, and Y.-M. Chu, "Some new Simpson-type inequalities for generalized p-convex function on fractal sets with applications," Advances in Difference Equations, vol. 2020, Article ID 2955-9, 1 page, 2020. 
[7] S. Rashid, M. A. Noor, K. I. Noor, F. Safdar, and Y.-M. Chu, "Hermite-hadamard type inequalities for the class of convex functions on time scale," Mathematics, vol. 7, no. 10, p. 956, 2019.

[8] S. I. Butt, M. Umar, S. Rashid, A. O. Akdemir, and Y.-M. Chu, "New Hermite-Jensen-Mercer-type inequalities via $\mathrm{k}$-fractional integrals," Advances in Difference Equations, vol. 2020, Article ID 03093-y, 1 page, 2020.

[9] S. Ihsan Butt, A. Kashuri, A. Kashuri, A. Aslam, and W. Gao, "Hermite-Jensen-Mercer type inequalities via $\Psi$-RiemannLiouville $k$-fractional integrals," AIMS Mathematics, vol. 5, no. 5, pp. 5193-5220, 2020.

[10] S. Zhao, S. I. Butt, W. Nazeer, J. Nasir, M. Umar, and Y. Liu, "Some Hermite-Jensen-Mercer type inequalities for $k$ Caputo-fractional derivatives and related results," Advances in Difference Equations, vol. 2020, Article ID 2693-y, 1 page, 2020.

[11] J. Pečarić, D. S. Mitrinovic, and A. M. Fink, Classical and New Inequalities in Analysis, pp. 1-2, Springer, Berlin, Germany, 1993.

[12] M. Adil Khan, S. Khan, I. Ullah, K. Ali Khan, and Y. M. Chu, "A novel approach to the Jensen gap through Taylor's theorem," Mathematical Methods in the Applied Sciences, vol. 44, no. 5, pp. 3324-3333, 2021.

[13] S. I. Butt, M. Klaričić Bakula, Đ. Pečarić, and J. Pečarić, "Jensen-grüss inequality and its applications for the zipfmandelbrot law," Mathematical Methods in the Applied Sciences, vol. 44, no. 2, pp. 1664-1673, 2021.

[14] N. Mehmood, S. I. Butt, D. Pečarić, and J. Pečarić, "Generalizations of cyclic refinements of Jensen's inequality by Lidstone's polynomial with applications in information theory," Journal of Mathematical Inequalities, vol. 14, no. 1, pp. 249-271, 2019.

[15] A. McD. Mercer, “A variant of Jensen's inequality," Journal of Inequalities in Pure and Applied Mathematics, vol. 4, no. 4, Article ID 23792-8_7, 2003.

[16] A. Matkovic, J. Pečarić, and I. Perić, "A variant of Jensens inequality of Mercer's type for operators with applications," Linear Algebra and Its Applications, vol. 418, pp. 551-564, 2006.

[17] M. Niezgoda, “A generalization of Mercer's result on convex functions," Nonlinear Analysis Forum, vol. 71, 2009.

[18] E. Anjidani and M. R. Changalvaiy, "Reverse jensen-mercer type operator inequalities," The Electronic Journal of Linear Algebra, vol. 31, pp. 87-99, 2016.

[19] H. R. Moradi and S. Furuichi, "Improvement and generalization of some Jensen-Mercer-type inequalities," Journal of Mathematical Inequalities, vol. 14, no. 2, pp. 377-383, 2020.

[20] M. A. Khan, Z. Husain, and Y. M. Chu, "New estimates for Csiszár divergence and zipf-mandelbrot entropy via jensen-mercer's inequality," Complexity, vol. 2020, Article ID 8928691, 8 pages, 2020.

[21] R. Khalil, M. Al Horani, A. Yousef, and M. Sababheh, "A new definition of fractional derivative," Journal of Computational and Applied Mathematics, vol. 264, pp. 65-70, 2014.

[22] R. Almeida, M. Guzowska, and T. Odzijewicz, "A remark on local fractional calculus and ordinary derivatives," Open Mathematics, vol. 14, no. 1, pp. 1122-1124, 2016.

[23] A. A. Kilbas, H. M. Srivastava, and J. J. Trujillo, "Theory and applications of fractional differential equations," Math. Studies, North-Holland, New York, NY, USA, 2006.

[24] K. S. Miller and B. Ross, An Introduction to Fractional Calculus and Fractional Differential Equations, A Wiley-
Interscience Publication, John Wiley and Sons, Inc., New York, NY, USA, 1993.

[25] E. Capelas de Oliveira and E. C. Oliveira, "On the $\psi$-Hilfer fractional derivative," Communications in Nonlinear Science and Numerical Simulation, vol. 60, pp. 72-91, 2018.

[26] J. Vanterler da Costa Sousa and E. Capelas de Oliveira, "A Gronwall inequality and the Cauchy-type problem by means of $\psi$-Hilfer operator," Differential Equations \& Applications, vol. 11, no. 1, pp. 87-106, 2019.

[27] H. Öğülmüş and M. Z. Sarikaya, "Hermite-Hadamard-Mercer type inequalities for fractional integrals," 2019, https:/www.researchgate.net/publication/ 337682540 .

[28] M. Kian and M. S. Moslehian, "Refinements of the operator jensen-mercer inequality," The Electronic Journal of Linear Algebra, vol. 26, pp. 742-753, 2013.

[29] Q. Kang, S. I. Butt, W. Nazeer, M. Nadeem, J. Nasir, and H. Yang, "New variant of Hermite-Jensen-Mercer type inequalities via Riemann-Liouville fractional integral operators," Jurnal Matematika, vol. 2020, Article ID 4303727, 14 pages, 2020.

[30] K. Liu, J. R. Wang, and D. O’Regan, “On the Hermite-Hadamard type inequality for $\psi$-Riemann-Liouville fractional integrals via convex functions," Journal of Inequalities and Applications, vol. 2019, Article ID 19-1982-1, 27 pages, 2019.

[31] M. Z. Sarikaya and H. Yildirim, "On Hermite-Hadamard type inequalities for Riemann-Liouville fractional integrals," Miskolc Mathematical Notes, vol. 17, no. 2, pp. 1049-1059, 2016.

[32] M. A. Noor and M. U. Awan, "Some integral inequalities for two kinds of convexities via fractional integrals," Transylvanian Journals of Mathematics and Mechanics, vol. 5, no. 2, pp. 129-136, 2013.

[33] M. Z. Sarikaya and N. Aktan, "On the generalization of some integral inequalities and their applications," Mathematical and Computer Modelling, vol. 54, no. 9-10, pp. 2175-2182, 2011.

[34] B. Bayraktar, "Some integral inequalities of Hermite-Hadamard type for differentable $(s, m)$-convex functions via fractional integrals," TWMS Journal of Applied and Engineering Mathematics, vol. 10, no. 3, pp. 625-637, 2020.

[35] G. N. Watson, A Treatise on the Theory of Bessel Functions, Cambridge University Press, Cambridge, UK, 1944.

[36] Y. L. Luke, The Special Functions and Their Approximations, vol. 1, Academic Press, Cambridge, MA, USA, 1969. 